\title{
An Alternative Approach to Integrable Discrete Nonlinear Schrödinger Equations
}

\author{
Francesco Demontis · Cornelis van der Mee
}

Received: 2 July 2012 / Accepted: 21 December 2012 / Published online: 19 January 2013

(C) Springer Science+Business Media Dordrecht 2013

\begin{abstract}
In this article we develop the direct and inverse scattering theory of a discrete matrix Zakharov-Shabat system with solutions $\boldsymbol{U}_{n}$ and $\boldsymbol{W}_{n}$. Contrary to the discretization scheme enacted by Ablowitz and Ladik, a central difference scheme is applied to the positional derivative term in the matrix Zakharov-Shabat system to arrive at a different discrete linear system. The major effect of the new discretization is that we no longer need the following two conditions in theories based on the Ablowitz-Ladik discretization: (a) invertibility of $I_{N}-\boldsymbol{U}_{n} \boldsymbol{W}_{n}$ and $I_{M}-\boldsymbol{W}_{n} \boldsymbol{U}_{n}$, and (b) $I_{N}-\boldsymbol{U}_{n} \boldsymbol{W}_{n}$ and $I_{M}-\boldsymbol{W}_{n} \boldsymbol{U}_{n}$ being nonzero multiples of the respective identity matrices $I_{N}$ and $I_{M}$.
\end{abstract}

Keywords Inverse scattering transform · Integrable discrete nonlinear Schroedinger equation · Marchenko method

\section{Introduction}

The system of integrable discrete nonlinear Schrödinger (IDNLS) equations

$$
\begin{gathered}
i \frac{d}{d \tau} \boldsymbol{U}_{n}=\boldsymbol{U}_{n+1}-2 \boldsymbol{U}_{n}+\boldsymbol{U}_{n-1}-\boldsymbol{U}_{n+1} \boldsymbol{W}_{n} \boldsymbol{U}_{n}-\boldsymbol{U}_{n} \boldsymbol{W}_{n} \boldsymbol{U}_{n-1}, \\
-i \frac{d}{d \tau} \boldsymbol{W}_{n}=\boldsymbol{W}_{n+1}-2 \boldsymbol{W}_{n}+\boldsymbol{W}_{n-1}-\boldsymbol{W}_{n+1} \boldsymbol{U}_{n} \boldsymbol{W}_{n}-\boldsymbol{W}_{n} \boldsymbol{U}_{n} \boldsymbol{W}_{n-1},
\end{gathered}
$$

where $n$ is an integer labeling "position" and $\boldsymbol{U}_{n}$ and $\boldsymbol{W}_{n}$ are $N \times M$ and $M \times N$ matrix functions depending on "time" $\tau \in \mathbb{R}$, has been studied extensively ever since the seminal papers [2-4] on the $N=M=1$ case, where the inverse scattering transform (IST) method to solve the initial-value problem for (1.1a), (1.1b) was first introduced. The spectral problem to which the nonlinear evolution equations (1.1a), (1.1b) were associated by means of the

F. Demontis · C. van der Mee ( $\bowtie)$

Dip. Matematica, Università di Cagliari, Viale Merello 92, 09123 Cagliari, Italy

e-mail: cornelis@krein.unica.it 
IST, is the discrete matrix Zakharov-Shabat system

$$
\boldsymbol{v}_{n+1}=\left(\begin{array}{cc}
z I_{N} & \boldsymbol{U}_{n} \\
\boldsymbol{W}_{n} & z^{-1} I_{M}
\end{array}\right) \boldsymbol{v}_{n}
$$

where $I_{N}$ is the identity matrix of order $N, z$ is a complex spectral parameter, and the potentials $\left\{\boldsymbol{U}_{n}\right\}_{n=-\infty}^{\infty}$ and $\left\{\boldsymbol{W}_{n}\right\}_{n=-\infty}^{\infty}$ satisfy the $\ell^{1}$-condition

$$
\sum_{n=-\infty}^{\infty}\left(\left\|\boldsymbol{U}_{n}\right\|+\left\|\boldsymbol{W}_{n}\right\|\right)<+\infty .
$$

The focusing case occurs if $\boldsymbol{W}_{n}=-\boldsymbol{U}_{n}^{\dagger}$ for each integer $n$, where the dagger denotes conjugate matrix transposition; the defocussing case occurs if $\boldsymbol{W}_{n}=\boldsymbol{U}_{n}$ for each integer $n$. The direct and inverse scattering theory for (1.2) was studied as early as 1981 [14, 15]. Comprehensive accounts of its scattering theory were given in [6] and [24]. In [6, 24] the matrix equations (1.1a), (1.1b) were studied in detail using the IST. In [1, 25] the defocussing $N=M=1$ problem was studied for potentials not vanishing as $n \rightarrow \pm \infty$.

When viewing the matrix IDNLS equation as a finite difference approximation of the matrix NLS equation, it has the same applications as the matrix NLS equation, namely electromagnetic wave propagation in nonlinear media [22, 27], surface waves on sufficiently deep waters [27], and signal propagation in optical fibers [16, 17]. Apart from that, the matrix IDNLS equation has applications to the dynamics of a discrete curve on an ultraspherical surface [12], the dynamics of triangulations of surfaces [18], and Hamiltonian flows [20, 23].

Equation (1.2) can be viewed as the finite difference approximation of the $(N+M)$ Zakharov-Shabat system in the position variable $x \in \mathbb{R}$, where the derivative with respect to $x$ is replaced by the forward difference term $\left[\boldsymbol{v}_{n+1}-\boldsymbol{v}_{n}\right] / h$, the potentials $\boldsymbol{u}(x)$ and $\boldsymbol{w}(x)$ are replaced by $\boldsymbol{U}_{n}=h \boldsymbol{u}(n h)$ and $\boldsymbol{W}_{n}=h \boldsymbol{w}(n h)$, and the spectral parameter $k \in \mathbb{R}$ is replaced by $z=e^{-i k h}=1-i k h+O\left(h^{2}\right)$. This forward difference scheme leads to the spectral problem (1.2) which lacks forward-backward symmetry and requires the assumption the square matrix of order $N+M$ describing the transition $\boldsymbol{v}_{n} \mapsto \boldsymbol{v}_{n+1}$ is invertible for each $n \in \mathbb{Z}$. In fact, the authors of $[6,24]$ found it useful to essentially "scalarize" the nonlinear evolution system (1.1a), (1.1b) by requiring the potentials $\left\{\boldsymbol{U}_{n}\right\}_{n=-\infty}^{\infty}$ and $\left\{\boldsymbol{W}_{n}\right\}_{n=-\infty}^{\infty}$ to also satisfy the condition

$$
\boldsymbol{U}_{n} \boldsymbol{W}_{n}=\boldsymbol{W}_{n} \boldsymbol{U}_{n}=c_{n} I_{N}, \quad n \in \mathbb{Z},
$$

where $N=M$ and, for each $n \in \mathbb{Z}, c_{n}(\neq 1)$ is an unknown complex number. Assuming (1.4), in the focusing case the scattering matrix, when multiplied by a weight matrix, appears to be symplectically unitary [10]. No weight factor is needed to prove the symplectic unitarity of the scattering matrix for the continuous-position Zakharov-Shabat system [6].

The next difficulty in solving the IDNLS system (1.1a), (1.1b) using the IST based on the discrete Zakharov-Shabat system (1.2) regards the Marchenko method. Although condition (1.4) is not required to derive the Marchenko equations [6, Eqs. (5.2.151) and (5.2.153)] and the "renormalized" Marchenko equations [6, Eqs. (5.2.158)], it is to be assumed in order to retrieve the IDNLS solution from the renormalized Marchenko solution. Without assuming condition (1.4), a priori information on the IDNLS solution is required. After the $N$-soliton and breather solutions to (1.1a), (1.1b) under condition (1.4) were derived before in terms of solutions to $N \times N$ linear systems [24, Eq. (3.43)], breather solutions were constructed before by using the Hirota method [7], and one and two soliton solutions were derived by 
various methods [5], an extensive family of IDNLS solutions in terms of triplets of matrices parametrizing the Marchenko kernel was derived in [10, 11].

In this article the apparent difficulty in solving the IDNLS system by using the IST based on the scattering theory for the discrete equation (1.2) is overcome by replacing (1.2) by the discrete matrix Zakharov-Shabat system

$$
\frac{\boldsymbol{v}_{n+1}(z)-\boldsymbol{v}_{n-1}(z)}{2 i h}=J\left(\begin{array}{cc}
\lambda I_{N} & \boldsymbol{u}_{n} \\
\boldsymbol{w}_{n} & \lambda I_{M}
\end{array}\right) \boldsymbol{v}_{n}(z),
$$

where $J=\operatorname{diag}\left(I_{N},-I_{M}\right),\left\{\boldsymbol{u}_{n}\right\}_{n=-\infty}^{\infty}$ and $\left\{\boldsymbol{w}_{n}\right\}_{n=-\infty}^{\infty}$ are potentials, $h>0$ is the discretization step size, $\lambda=\left(z-z^{-1}\right) / 2 i h$ is a spectral parameter, and the $\ell^{1}$-condition (1.3) is assumed. Thus (1.5) arises from the matrix Zakharov-Shabat system by a central finite difference scheme. The essential spectrum coincides with $\lambda \in\left[-h^{-1}, h^{-1}\right]$ and with $z \in \mathbb{T}, \mathbb{T}$ being the unit circle. As for (1.2), the focusing case occurs if $\boldsymbol{w}_{n}=-\boldsymbol{u}_{n}^{\dagger}$ for each $n \in \mathbb{Z}$; the defocussing case occurs if $\boldsymbol{w}_{n}=\boldsymbol{u}_{n}^{\dagger}$ for each $n \in \mathbb{Z}$.

Equation (1.5) as the linear counterpart to (1.1a), (1.1b) has none of the problems inherent in choosing (1.2) as its linear counterpart. Since (1.5) can be written in the form

$$
\left(\begin{array}{c}
\boldsymbol{v}_{n+1}(z) \\
\boldsymbol{v}_{n}(z)
\end{array}\right)=\left(\begin{array}{cccc}
\lambda I_{N} & \boldsymbol{u}_{n} & I_{N} & 0_{N, M} \\
-\boldsymbol{w}_{n} & -\lambda I_{M} & 0_{M, N} & I_{M} \\
I_{N} & 0_{N, M} & 0_{N, N} & 0_{N, M} \\
0_{M, N} & I_{M} & 0_{M, N} & 0_{M, M}
\end{array}\right)\left(\begin{array}{c}
\boldsymbol{v}_{n}(z) \\
\boldsymbol{v}_{n-1}(z)
\end{array}\right)
$$

where the square coupling matrix of order $N+M$ is nonsingular, we do not need to assume the invertibility of $I_{N}-\boldsymbol{u}_{n} \boldsymbol{w}_{n}$ and $I_{M}-\boldsymbol{w}_{n} \boldsymbol{u}_{n}$ for each $n \in \mathbb{Z}$ in order to compute $\boldsymbol{v}_{n}(z)$ from $\boldsymbol{v}_{n \pm 1}(z)$ and $\boldsymbol{v}_{n \pm 2}(z)$ uniquely. Furthermore, getting the potentials from the Marchenko equations proceeds in very much the same way as for the continuous Zakharov-Shabat system and does not require renormalization of the Marchenko kernel. Also, in the focusing case the scattering matrix $S(z)$ is $J$-unitary in the sense that $S(z)^{-1}=J S(z)^{\dagger} J$; in the defocussing case $\boldsymbol{S}(z)$ is a unitary matrix.

Although we have given a full description of the IST to solve the matrix IDNLS system (1.1a), (1.1b) using the scattering theory of (1.5), we have not been able to furnish a Lax pair $\{L, A\}$, where

$$
(L \boldsymbol{v})_{n}=J \frac{\boldsymbol{v}_{n+1}-\boldsymbol{v}_{n-1}}{2 i h}-\left(\begin{array}{cc}
0_{N, N} & \boldsymbol{u}_{n} \\
\boldsymbol{w}_{n} & 0_{M, M}
\end{array}\right) \boldsymbol{v}_{n}
$$

is the operator rewrite of (1.5) and the usual compatibility condition $L_{t}+L A-A L=0$ reduces to the matrix IDNLS system (1.1a), (1.1b). We have also not found an AKNS pair $\left\{\boldsymbol{X}_{n}, \boldsymbol{T}_{n}\right\}$ of matrices depending on $\lambda$ such that $\boldsymbol{V}_{n+1}=\boldsymbol{X}_{n} \boldsymbol{V}_{n}$ is the rewrite of (1.6), $\left[\boldsymbol{V}_{n}\right]_{t}=$ $\boldsymbol{T}_{n} \boldsymbol{V}_{n}$, and the compatibility condition $\left[\boldsymbol{X}_{n}\right]_{t}+\boldsymbol{X}_{n} \boldsymbol{T}_{n}-\boldsymbol{T}_{n+1} \boldsymbol{X}_{n}=0$ reduces to the matrix IDNLS system. Such a pair, where $\boldsymbol{v}_{n+1}=\boldsymbol{X}_{n} \boldsymbol{v}_{n}$ is the rewrite of (1.2), [ $\left.\boldsymbol{v}_{n}\right]_{t}=\boldsymbol{T}_{n} \boldsymbol{v}_{n}$, and the preceding compatibility condition reduces to the matrix IDNLS system, is known (cf. [6, Eq. (5.1.2)] and [24, Eqs. (2.3)-(2.4)]). Finding at least one such pair involving a rewrite of (1.5) would be most desirable, in spite of their redundancy in formulating the IST and solving the initial-value problem for (1.1a), (1.1b).

The time evolution of the reflection coefficients, norming constants, and Marchenko kernels associated with the discrete Zakharov-Shabat system (1.2) leading to IDNLS solutions has been explained in detail in [6], also without assuming condition (1.4). The renormalized 
Marchenko equations given in $[6,10,24]$ have the form

$$
\begin{aligned}
& \overline{\boldsymbol{\kappa}}(n, j ; \tau)+\left(\begin{array}{c}
0_{N, N} \\
I_{N}
\end{array}\right) \boldsymbol{F}(n+j ; \tau)+\sum_{j^{\prime}=n+1}^{\infty} \boldsymbol{\kappa}\left(n, j^{\prime} ; \tau\right) \boldsymbol{F}\left(j+j^{\prime} ; \tau\right)=0_{2 N, N}, \\
& \boldsymbol{\kappa}(n, j ; \tau)+\left(\begin{array}{c}
I_{N} \\
0_{N, N}
\end{array}\right) \overline{\boldsymbol{F}}(n+j ; \tau)+\sum_{j^{\prime}=n+1}^{\infty} \overline{\boldsymbol{\kappa}}\left(n, j^{\prime} ; \tau\right) \overline{\boldsymbol{F}}\left(j+j^{\prime} ; \tau\right)=0_{2 N, N},
\end{aligned}
$$

where $j \geq n+1$ and the Marchenko kernels $\boldsymbol{F}$ and $\overline{\boldsymbol{F}}$ are given. On the other hand, within the scattering theory of (1.2) it is possible to derive alternative Marchenko equations satisfied for $j \leq n-1$, where the summation index $j^{\prime}$ runs from $-\infty$ to $n-1$. These equations can be derived without assuming condition (1.4) and coincide exactly with the Marchenko equations $(5.4 \mathrm{c})-(5.4 \mathrm{~d})$ below [11]. The crux of the matter is that the Marchenko equations (5.4c)-(5.4d) below will also be derived within the scattering theory of (1.5). Thus the same Marchenko equations and the same formulas to pass from the Marchenko solutions to the potentials are derived within the scattering theories of two different linear spectral problems, (1.2) and (1.5). Consequently, the same time varying Marchenko solutions are found and hence the same time varying potentials when departing from the same initial potentials. This can only mean that we have in fact found an IST method for solving the IDNLS system (1.1a), (1.1b) based on the scattering theory of (1.5) which, quite obviously, does not require assuming condition (1.4).

If we allow the potentials to be time dependent such that (1.1a), (1.1b) are satisfied, then the Marchenko kernels $\boldsymbol{F}(n ; \tau)$ and $\overline{\boldsymbol{F}}(n ; \tau)$ satisfy the evolution equations

$$
\begin{aligned}
i \frac{d}{d \tau} \boldsymbol{F}(n ; \tau) & =\boldsymbol{F}(n+2 ; \tau)-2 \boldsymbol{F}(n ; \tau)+\boldsymbol{F}(n-2 ; \tau), \\
-i \frac{d}{d \tau} \overline{\boldsymbol{F}}(n ; \tau) & =\overline{\boldsymbol{F}}(n+2 ; \tau)-2 \overline{\boldsymbol{F}}(n ; \tau)+\overline{\boldsymbol{F}}(n-2 ; \tau) .
\end{aligned}
$$

For $N=M=1$, Eqs. (1.8a), (1.8b) may be considered as the equations of motion of a Hamiltonian system with Hamiltonian

$$
\mathcal{H}=i \sum_{n \text { odd }}[\boldsymbol{F}(n+2 ; \tau)-\boldsymbol{F}(n ; \tau)][\overline{\boldsymbol{F}}(n+2 ; \tau)-\overline{\boldsymbol{F}}(n ; \tau)],
$$

where $\{\boldsymbol{F}(2 s+1 ; \tau)\}_{s=-\infty}^{\infty}$ are the coordinates and $\{\overline{\boldsymbol{F}}(2 s+1 ; \tau)\}_{s=-\infty}^{\infty}$ are the corresponding momenta. The inverse scattering transform can then be viewed as a canonical transformation between Hamiltonian systems.

The explicit matrix IDNLS solutions obtained in [11] by using matrix triplets to parametrize the Marchenko kernels do not necessarily satisfy condition (1.4) nor the condition of invertibility of $I_{N}-\boldsymbol{u}_{n} \boldsymbol{w}_{n}$. In retrospect, this is not surprising, because these solutions can be obtained by the IST method based on the linear spectral problem (1.5). In any case, the explicit solutions that do satisfy condition (1.4), are included in our description. It was also shown in [11] that the matrix IDNLS solutions obtained converge to known explicit solutions of the matrix NLS equation as the discretization step $h$ vanishes. We shall therefore not present these solutions in this article.

Let us now describe the contents of the subsequent sections. In Sect. 2 we introduce the Jost solutions and derive their continuity and analyticity properties. In Sect. 3 we introduce the transition coefficients translating the $n \rightarrow+\infty$ asymptotics of the Jost solutions into 
their $n \rightarrow-\infty$ asymptotics or vice versa and derive their continuity and analyticity properties. In Sect. 4 we define the reflection and transmission coefficients and introduce the scattering matrix. In Sect. 5 we derive the Marchenko integral equations and indicate how the potentials are to be found from their solutions. Appendix A contains estimates on the discrete Fourier transforms of the Jost solutions. In Appendix B we use parity symmetry to decouple the Marchenko equations as much as possible.

\section{Jost Solutions}

In this section we define the Jost solutions for the discrete matrix Zakharov-Shabat system (1.5) and derive their continuity and analyticity properties.

For $z \in \mathbb{T}$ we define the Jost solutions $\phi_{n}(z), \bar{\phi}_{n}(z), \psi_{n}(z)$, and $\bar{\psi}_{n}(z)$ as those solutions of (1.5) that satisfy the asymptotic conditions

$$
\begin{array}{ll}
\phi_{n}(z) \sim z^{n}\left(\begin{array}{c}
I_{N} \\
0_{M, N}
\end{array}\right), & \bar{\phi}_{n}(z) \sim z^{-n}\left(\begin{array}{c}
0_{N, M} \\
I_{M}
\end{array}\right), \quad \text { as } n \rightarrow-\infty, \\
\psi_{n}(z) \sim z^{-n}\left(\begin{array}{c}
0_{N, M} \\
I_{M}
\end{array}\right), & \bar{\psi}_{n}(z) \sim z^{n}\left(\begin{array}{c}
I_{N} \\
0_{M, N}
\end{array}\right), \quad \text { as } n \rightarrow+\infty .
\end{array}
$$

Their unique existence will be proven below [cf. Proposition 2.1]. We observe that for $0 \neq$ $z \in \mathbb{C}$ the Jost matrices $\left(\phi_{n}(z) \bar{\phi}_{n}(z)\right)$ and $\left(\bar{\psi}_{n}(z) \psi_{n}(z)\right)$ approach nonsingular matrices as $n \rightarrow+\infty$ and $n \rightarrow-\infty$, respectively.

Equation (1.5) displays parity symmetry: If $\boldsymbol{v}_{n}(z)$ is a solution to $(1.5)$, then $\tilde{\boldsymbol{v}}_{n}(z)=$ $(-1)^{n} J v_{n}(-z) J$ is also a solution to (1.5). Thus the Jost solutions have the parity properties:

$$
\begin{aligned}
& \left(\bar{\psi}_{n}(z) \quad \psi_{n}(z)\right)=(-1)^{n} J\left(\bar{\psi}_{n}(-z) \quad \psi_{n}(-z)\right) J, \\
& \left(\phi_{n}(z) \quad \bar{\phi}_{n}(z)\right)=(-1)^{n} J\left(\phi_{n}(-z) \quad \bar{\phi}_{n}(-z)\right) J .
\end{aligned}
$$

Equation (1.5) also displays inversion symmetry: If $\boldsymbol{v}_{n}(z)$ is a solution to (1.5), then $\tilde{\boldsymbol{v}}_{n}(z)=(-1)^{n} J \boldsymbol{v}_{n}(z) J$ is a solution to the system obtained from (1.5) by replacing $\lambda$ by $-\lambda$. Inversion symmetry implies $(2.2 \mathrm{a}),(2.2 \mathrm{~b})$.

Let us now introduce the following Faddeev condition on the potentials:

$$
\sum_{n=-\infty}^{\infty}(1+|n|)\left(\left\|\boldsymbol{u}_{n}\right\|+\left\|\boldsymbol{w}_{n}\right\|\right)<+\infty .
$$

We can then derive the following existence result for Jost solutions, reminiscent of the result valid for the Schrödinger equation on the line [8, 13, 19].

Proposition 2.1 (Existence of Jost solutions) Suppose either potential $\left\{\boldsymbol{u}_{n}\right\}_{n=-\infty}^{\infty}$ and $\left\{\boldsymbol{w}_{n}\right\}_{n=-\infty}^{\infty}$ satisfies the $\ell^{1}$-condition (1.3). Then for $\pm i \neq z \in \mathbb{T}$ there exist unique Jost solutions $\psi_{n}(z), \bar{\psi}_{n}(z), \phi_{n}(z)$, and $\bar{\phi}_{n}(z)$ of the discrete matrix Zakharov-Shabat system (1.5) that satisfy (2.1a), (2.1b). Moreover, if these potentials satisfy the Faddeev condition (2.3), then for $z \in \mathbb{T}$ there exist unique Jost solutions $\psi_{n}(z), \bar{\psi}_{n}(z), \phi_{n}(z)$, and $\bar{\phi}_{n}(z)$ of the discrete matrix Zakharov-Shabat system (1.5) that satisfy (2.1a), (2.1b). 
Proof Let us define the Faddeev functions by

$$
\begin{aligned}
\boldsymbol{M}_{n}(z) & =z^{-n} \phi_{n}(z), & \overline{\boldsymbol{M}}_{n}(z) & =z^{n} \bar{\phi}_{n}(z), \\
\boldsymbol{N}_{n}(z) & =z^{n} \psi_{n}(z), & \overline{\boldsymbol{N}}_{n}(z) & =z^{-n} \bar{\psi}_{n}(z) .
\end{aligned}
$$

Observing that $\lambda J=\left(\boldsymbol{Z}-\boldsymbol{Z}^{-1}\right) / 2 i h$, we put

$$
\boldsymbol{V}_{n}=\left(\begin{array}{cc}
0_{N, N} & \boldsymbol{u}_{n} \\
\boldsymbol{w}_{n} & 0_{M, M}
\end{array}\right)
$$

Then, writing $\boldsymbol{v}_{n}(z)=\boldsymbol{m}_{n}(z) \boldsymbol{Z}^{n}$ for a solution of (1.5), we get

$$
\left[\boldsymbol{m}_{n+1}(z) \boldsymbol{Z}-\boldsymbol{Z m}_{n}(z)\right]=-\boldsymbol{Z}^{-1}\left[\boldsymbol{m}_{n}(z) \boldsymbol{Z}-\boldsymbol{Z}_{\boldsymbol{m}_{n-1}}(z)\right] \boldsymbol{Z}^{-1}+2 i h J \boldsymbol{V}_{n} \boldsymbol{m}_{n}(z) .
$$

Putting

$$
\boldsymbol{\Delta}_{n}(z)=\boldsymbol{m}_{n}(z) \boldsymbol{Z}-\boldsymbol{Z} \boldsymbol{m}_{n-1}(z),
$$

we get by iteration

$$
\begin{aligned}
& \boldsymbol{\Delta}_{n}(z)=(-1)^{p} \boldsymbol{Z}^{p} \boldsymbol{\Delta}_{n+p}(z) \boldsymbol{Z}^{p}+2 i h J \sum_{\tau=0}^{p-1}(-1)^{\tau} \boldsymbol{Z}^{\tau+1} \boldsymbol{V}_{n+\tau} \boldsymbol{m}_{n+\tau}(z) \boldsymbol{Z}^{\tau+1}, \\
& \boldsymbol{\Delta}_{n}(z)=(-1)^{p} \boldsymbol{Z}^{-p} \boldsymbol{\Delta}_{n-p}(z) \boldsymbol{Z}^{-p}-2 i h J \sum_{\tau=0}^{p-1}(-1)^{\tau} \boldsymbol{Z}^{-\tau} \boldsymbol{V}_{n-\tau-1} \boldsymbol{m}_{n-\tau-1}(z) \boldsymbol{Z}^{-\tau} .
\end{aligned}
$$

Now note that, as $p \rightarrow+\infty, \boldsymbol{\Delta}_{n+p}(z)$ vanishes for $\boldsymbol{v}_{n}(z)=\left(\bar{\psi}_{n}(z) \psi_{n}(z)\right)$ and $\boldsymbol{\Delta}_{n-p}(z)$ vanishes for $\boldsymbol{v}_{n}(z)=\left(\phi_{n}(z) \bar{\phi}_{n}(z)\right)$, while $\left\|\boldsymbol{Z}^{ \pm p}\right\|=1$. Consequently, we obtain

$$
\begin{aligned}
& \boldsymbol{m}_{n+1}(z)=\boldsymbol{Z}_{n}(z) \boldsymbol{Z}^{-1}+2 i h J \sum_{\tau=0}^{\infty}(-1)^{\tau} \boldsymbol{Z}^{\tau+1} \boldsymbol{V}_{n+\tau+1} \boldsymbol{m}_{n+\tau+1}(z) \boldsymbol{Z}^{\tau}, \\
& \boldsymbol{m}_{n-1}(z)=\boldsymbol{Z}^{-1} \boldsymbol{m}_{n}(z) \boldsymbol{Z}+2 i h J \sum_{\tau=0}^{\infty}(-1)^{\tau} \boldsymbol{Z}^{-\tau-1} \boldsymbol{V}_{n-\tau-1} \boldsymbol{m}_{n-\tau-1}(z) \boldsymbol{Z}^{-\tau},
\end{aligned}
$$

respectively.

Let us now solve the linear difference equation system

$$
\boldsymbol{m}_{n+1}(z) \boldsymbol{Z}-\boldsymbol{Z} \boldsymbol{m}_{n}(z)=\boldsymbol{F}_{n}^{+}(z), \quad \boldsymbol{m}_{n}(z) \boldsymbol{Z}-\boldsymbol{Z}_{n-1}(z)=\boldsymbol{F}_{n-1}^{-}(z),
$$

for given inhomogeneous term $\boldsymbol{F}_{n}^{ \pm}(z)$ under the asymptotic condition that $\boldsymbol{m}_{n}(z) \rightarrow I_{N+M}$ as $n \rightarrow \pm \infty$, respectively. We get by iteration

$$
\begin{aligned}
& \boldsymbol{m}_{n}(z)=\boldsymbol{Z}^{-p} \boldsymbol{m}_{n+p}(z) \boldsymbol{Z}^{p}-\sum_{\tau=0}^{p-1} \boldsymbol{Z}^{-\tau-1} \boldsymbol{F}_{n+\tau}^{+}(z) \boldsymbol{Z}^{\tau}, \\
& \boldsymbol{m}_{n}(z)=\boldsymbol{Z}^{p} \boldsymbol{m}_{n-p}(z) \boldsymbol{Z}^{-p}+\sum_{\tau=0}^{p-1} \boldsymbol{Z}^{\tau} \boldsymbol{F}_{n-\tau-1}^{-}(z) \boldsymbol{Z}^{-\tau-1} .
\end{aligned}
$$


Using that $\boldsymbol{m}_{n \pm p}(z)$ tend to diagonal matrices which evidently commute with $\boldsymbol{Z}$, we take the limit as $p \rightarrow+\infty$ and get

$$
\begin{aligned}
\left(\overline{\boldsymbol{N}}_{n}(z) \quad \boldsymbol{N}_{n}(z)\right)= & I_{N+M}-\sum_{\hat{s}=0}^{\infty} \boldsymbol{Z}^{-\hat{s}-1} \boldsymbol{F}_{n+\hat{s}}^{+}(z) \boldsymbol{Z}^{\hat{s}} \\
= & I_{N+M}-2 i h J \sum_{s=0}^{\infty}\left(\sum_{\tau=0}^{s}(-1)^{\tau} \boldsymbol{Z}^{-s+2 \tau}\right) \\
& \times \boldsymbol{V}_{n+s+1}\left(\overline{\boldsymbol{N}}_{n+s+1}(z) \quad \boldsymbol{N}_{n+s+1}(z)\right) \boldsymbol{Z}^{s+1}, \\
\left(\boldsymbol{M}_{n}(z) \quad \overline{\boldsymbol{M}}_{n}(z)\right)= & I_{N+M}+\sum_{\hat{s}=0}^{\infty} \boldsymbol{Z}^{\hat{s}} \boldsymbol{F}_{n-\hat{s}-1}^{-}(z) \boldsymbol{Z}^{-\hat{s}-1} \\
= & I_{N+M}-2 i h J \sum_{s=0}^{\infty}\left(\sum_{\tau=0}^{s}(-1)^{\tau} \boldsymbol{Z}^{s-2 \tau}\right) \\
& \times \boldsymbol{V}_{n-s-1}\left(\boldsymbol{M}_{n-s-1}(z) \quad \overline{\boldsymbol{M}}_{n-s-1}(z)\right) \boldsymbol{Z}^{-s-1} .
\end{aligned}
$$

Now observe that

$$
\begin{aligned}
\sum_{\tau=0}^{s}(-1)^{\tau} \boldsymbol{Z}^{-s+2 \tau} & = \begin{cases}{\left[\boldsymbol{Z}^{-s}+(-1)^{s} \boldsymbol{Z}^{s+2}\right]\left[I_{N+M}+\boldsymbol{Z}^{2}\right]^{-1},} & z \neq \pm i, \\
(s+1)( \pm i)^{-s} I_{N+M}, & z= \pm i\end{cases} \\
\sum_{\tau=0}^{s}(-1)^{\tau} \boldsymbol{Z}^{s-2 \tau} & = \begin{cases}{\left[\boldsymbol{Z}^{s+2}+(-1)^{s} \boldsymbol{Z}^{-s}\right]\left[I_{N+M}+\boldsymbol{Z}^{2}\right]^{-1},} & z \neq \pm i, \\
(s+1)( \pm i)^{s} I_{N+M}, & z= \pm i,\end{cases}
\end{aligned}
$$

where we have used well-known expressions for partial sums of geometric series. Using (2.6a), (2.6b) we easily prove that $(2.4 \mathrm{a}),(2.4 \mathrm{~b})$ are uniquely solvable by iteration, because of the estimate (1.3) used uniformly in $z$ on compact subsets of $\mathbb{T} \backslash\{ \pm i\}$ and the estimate (2.3) used uniformly in $z \in \mathbb{T}$. This completes the proof.

\section{Writing}

$$
\boldsymbol{F}^{\mathrm{up}}=\left(\begin{array}{ll}
I_{N} & 0_{N, M}
\end{array}\right) \boldsymbol{F}, \quad \boldsymbol{F}^{\mathrm{dn}}=\left(\begin{array}{ll}
0_{M, N} & I_{M}
\end{array}\right) \boldsymbol{F},
$$

for any matrix $\boldsymbol{F}$ having $N+M$ rows, we now write (2.5a), (2.5b) as the following eight Volterra equations:

$$
\begin{aligned}
& \overline{\boldsymbol{N}}_{n}^{\mathrm{up}}(z)=I_{N}-2 i h z \sum_{j=n+1}^{\infty}\left(\sum_{\tau=0}^{j-n-1}(-1)^{\tau} z^{2 \tau}\right) \boldsymbol{u}_{j} \overline{\boldsymbol{N}}_{j}^{\mathrm{dn}}(z), \\
& \overline{\boldsymbol{N}}_{n}^{\mathrm{dn}}(z)=2 i h z \sum_{j=n+1}^{\infty}\left(\sum_{\tau=0}^{j-n-1}(-1)^{\tau} z^{2(j-n-\tau-1)}\right) \boldsymbol{w}_{j} \overline{\boldsymbol{N}}_{j}^{\mathrm{up}}(z), \\
& \boldsymbol{N}_{n}^{\mathrm{up}}(z)=-2 i h z^{-1} \sum_{j=n+1}^{\infty}\left(\sum_{\tau=0}^{j-n-1}(-1)^{\tau} z^{2(\tau+n+1-j)}\right) \boldsymbol{u}_{j} \boldsymbol{N}_{j}^{\mathrm{dn}}(z),
\end{aligned}
$$




$$
\begin{aligned}
& \boldsymbol{N}_{n}^{\mathrm{dn}}(z)=I_{M}+2 i h z^{-1} \sum_{j=n+1}^{\infty}\left(\sum_{\tau=0}^{j-n-1}(-1)^{\tau} z^{-2 \tau}\right) \boldsymbol{w}_{j} \boldsymbol{N}_{j}^{\mathrm{up}}(z), \\
& \boldsymbol{M}_{n}^{\mathrm{up}}(z)=I_{N}-2 i h z^{-1} \sum_{j=-\infty}^{n-1}\left(\sum_{\tau=0}^{n-j-1}(-1)^{\tau} z^{-2 \tau}\right) \boldsymbol{u}_{j} \boldsymbol{M}_{j}^{\mathrm{dn}}(z), \\
& \boldsymbol{M}_{n}^{\mathrm{dn}}(z)=2 i h z^{-1} \sum_{j=-\infty}^{n-1}\left(\sum_{\tau=0}^{n-j-1}(-1)^{\tau} z^{2(\tau-n+j+1)}\right) \boldsymbol{w}_{j} \boldsymbol{M}_{j}^{\mathrm{up}}(z), \\
& \overline{\boldsymbol{M}}_{n}^{\mathrm{up}}(z)=-2 i h z \sum_{j=-\infty}^{n-1}\left(\sum_{\tau=0}^{n-j-1}(-1)^{\tau} z^{2(n-j-\tau-1)}\right) \boldsymbol{u}_{j} \overline{\boldsymbol{M}}_{j}^{\mathrm{dn}}(z), \\
& \overline{\boldsymbol{M}}_{n}^{\mathrm{dn}}(z)=I_{M}+2 i h z \sum_{j=-\infty}^{n-1}\left(\sum_{\tau=0}^{n-j-1}(-1)^{\tau} z^{2 \tau}\right) \boldsymbol{w}_{j} \overline{\boldsymbol{M}}_{j}^{\mathrm{up}}(z) .
\end{aligned}
$$

Under the Faddeev condition (2.3), we easily prove that $\overline{\boldsymbol{N}}_{n}^{\mathrm{up}}(z), \overline{\boldsymbol{N}}_{n}^{\mathrm{dn}}(z), \overline{\boldsymbol{M}}_{n}^{\mathrm{up}}(z)$, and $\overline{\boldsymbol{M}}_{n}^{\mathrm{dn}}(z)$ are continuous in $|z| \leq 1$, are analytic in $|z|<1$, and behave as $I_{N}+O\left(z^{2}\right), O(z)$, $O(z)$, and $I_{M}+O\left(z^{2}\right)$ as $z \rightarrow 0$. Under the same condition we prove that $N_{n}^{\text {up }}(z), N_{n}^{\mathrm{dn}}(z)$, $\boldsymbol{M}_{n}^{\text {up }}(z)$, and $\boldsymbol{M}_{n}^{\mathrm{dn}}(z)$ are continuous in $|z| \geq 1$, are analytic in $|z|>1$, and behave as $O\left(z^{-1}\right)$, $I_{M}+O\left(z^{-2}\right), I_{N}+O\left(z^{-2}\right)$, and $O\left(z^{-1}\right)$ as $|z| \rightarrow+\infty$. If we only assume (1.3), certain Faddeev function blocks may have discontinuities as $z \rightarrow \pm i$ from the appropriate left or right-hand side of the unit circle.

Let us denote by $\mathcal{W}^{p, q}$ the set of all sums of absolutely convergent Fourier series whose coefficients are complex $p \times q$ matrices. Then $\mathcal{W}^{p, q}$ has a natural norm with respect to which it is a complex Banach space, namely

$$
\|\boldsymbol{Y}\|=\sum_{n=-\infty}^{\infty}\left\|Y_{n}\right\|, \quad \boldsymbol{Y}(z)=\sum_{n=-\infty}^{\infty} z^{n} Y_{n} .
$$

It is a complex Banach algebra if $p=q$. By a famous result by Wiener [26], this algebra is inverse closed in the sense that $Y^{-1} \in \mathcal{W}^{p, p}$ whenever $Y \in \mathcal{W}^{p, p}$ and $\operatorname{det} W(z) \neq 0$ for each $z \in \mathbb{T}$.

By $\mathcal{W}_{+}^{p, q}$ we denote the subspace of $\mathcal{W}^{p, q}$ consisting of those sums of Fourier series having an analytic continuation to the unit disk. On the other hand, by $\mathcal{W}_{-}^{p, q}$ we denote the subspace of $\mathcal{W}^{p, q}$ consisting of the sums of those Fourier series having an analytic continuation to the exterior of the unit disk, the point at infinity included.

In order to prove that, under condition (2.3), $\boldsymbol{M}_{n}(z), \overline{\boldsymbol{M}}_{n}(z), \overline{\boldsymbol{N}}_{n}(z)$, and $\boldsymbol{N}_{n}(z)$ belong to $\mathcal{W}_{+}^{N+M, N}, \mathcal{W}_{-}^{N+M, M}, \mathcal{W}_{-}^{N+M, N}$, and $\mathcal{W}_{+}^{N+M, M}$, respectively, we put

$$
\begin{array}{lll}
\overline{\boldsymbol{N}}_{n}^{\mathrm{up}}(z)=\sum_{j=n}^{\infty} z^{j-n} \overline{\boldsymbol{K}}^{\mathrm{up}}(n, j), & \overline{\boldsymbol{N}}_{n}^{\mathrm{dn}}(z)=\sum_{j=n}^{\infty} z^{j-n} \overline{\boldsymbol{K}}^{\mathrm{dn}}(n, j), \\
\boldsymbol{N}_{n}^{\mathrm{up}}(z)=\sum_{j=n}^{\infty} z^{n-j} \boldsymbol{K}^{\mathrm{up}}(n, j), & \boldsymbol{N}_{n}^{\mathrm{dn}}(z)=\sum_{j=n}^{\infty} z^{n-j} \boldsymbol{K}^{\mathrm{dn}}(n, j), \\
\boldsymbol{M}_{n}^{\mathrm{up}}(z)=\sum_{j=-\infty}^{n} z^{j-n} \boldsymbol{L}^{\mathrm{up}}(n, j), & \boldsymbol{M}_{n}^{\mathrm{dn}}(z)=\sum_{j=-\infty}^{n} z^{j-n} \boldsymbol{L}^{\mathrm{dn}}(n, j),
\end{array}
$$




$$
\overline{\boldsymbol{M}}_{n}^{\mathrm{up}}(z)=\sum_{j=-\infty}^{n} z^{n-j} \overline{\boldsymbol{L}}^{\mathrm{up}}(n, j), \quad \overline{\boldsymbol{M}}_{n}^{\mathrm{dn}}(z)=\sum_{j=-\infty}^{n} z^{n-j} \overline{\boldsymbol{L}}^{\mathrm{dn}}(n, j),
$$

where the Fourier coefficients are to be determined. Then obviously

$$
\begin{array}{ll}
\overline{\boldsymbol{K}}^{\text {up }}(n, n)=I_{N}, & \overline{\boldsymbol{K}}^{\mathrm{dn}}(n, n)=0_{M, N}, \\
\boldsymbol{K}^{\text {up }}(n, n)=0_{N, M}, & \boldsymbol{K}^{\mathrm{dn}}(n, n)=I_{M}, \\
\boldsymbol{L}^{\text {up }}(n, n)=I_{N}, & \boldsymbol{L}^{\mathrm{dn}}(n, n)=0_{M, N}, \\
\overline{\boldsymbol{L}}^{\text {up }}(n, n)=0_{N, M}, & \overline{\boldsymbol{L}}^{\mathrm{dn}}(n, n)=I_{M} .
\end{array}
$$

Let us now prove that the Faddeev functions belong to some $\mathcal{W}_{ \pm}^{p, q}$.

Proposition 2.2 (Jost solutions as Fourier sums) There are matrices $\boldsymbol{K}(n, j)$ and $\overline{\boldsymbol{K}}(n, j)$ satisfying

$$
\sum_{j=n}^{\infty}(\|\boldsymbol{K}(n, j)\|+\|\bar{K}(n, j)\|)<+\infty
$$

such that

$$
\psi_{n}(z)=\sum_{j=n}^{\infty} z^{-j} \boldsymbol{K}(n, j), \quad \bar{\psi}_{n}(z)=\sum_{j=n}^{\infty} z^{j} \overline{\boldsymbol{K}}(n, j) .
$$

Analogously, there exist matrices $\boldsymbol{L}(n, j)$ and $\overline{\mathbf{L}}(n, j)$ satisfying

$$
\sum_{j=-\infty}^{n}(\|\boldsymbol{L}(n, j)\|+\|\overline{\boldsymbol{L}}(n, j)\|)<+\infty
$$

such that

$$
\phi_{n}(z)=\sum_{j=-\infty}^{n} z^{j} \boldsymbol{L}(n, j), \quad \bar{\phi}_{n}(z)=\sum_{j=-\infty}^{n} z^{-j} \overline{\boldsymbol{L}}(n, j)
$$

Proof We apply (2.8a)-(2.8d) to convert (2.7a)-(2.7h) into the following identities:

$$
\begin{aligned}
& \overline{\boldsymbol{K}}^{\mathrm{up}}(n, j)=-2 i h \sum_{\tau=0}^{[(j-n-1) / 2]}(-1)^{\tau} \boldsymbol{u}_{j} \overline{\boldsymbol{K}}^{\mathrm{dn}}(j, 2 j-2 \tau-n-1), \\
& \overline{\boldsymbol{K}}^{\mathrm{dn}}(n, j)=2 i h \sum_{\tau=0}^{[(j-n-1) / 2]}(-1)^{j-n-\tau-1} \boldsymbol{w}_{j} \overline{\boldsymbol{K}}^{\mathrm{up}}(j, 2 j-2 \tau-n-1), \\
& \boldsymbol{K}^{\mathrm{up}}(n, j)=-2 i h \sum_{\tau=0}^{[(j-n-1) / 2]}(-1)^{j-n-\tau-1} \boldsymbol{u}_{j} \boldsymbol{K}^{\mathrm{dn}}(j, 2 j-2 \tau-n-1), \\
& \boldsymbol{K}^{\mathrm{dn}}(n, j)=2 i h \sum_{\tau=0}^{[(j-n-1) / 2]}(-1)^{\tau} \boldsymbol{w}_{j} \boldsymbol{K}^{\mathrm{up}}(j, 2 j-2 \tau-n-1),
\end{aligned}
$$


where $j \geq n+1$ and $[x]=\max \{n \in \mathbb{Z}: n \leq x\}$. Using (2.9a) and (2.9b) we get

$$
\overline{\boldsymbol{K}}^{\mathrm{dn}}(n, n+1)=2 i h \boldsymbol{w}_{n+1}, \quad \boldsymbol{K}^{\mathrm{up}}(n, n+1)=-2 i h \boldsymbol{u}_{n+1},
$$

as well as $\overline{\boldsymbol{K}}^{\text {up }}(n, n+1)=0_{N, N}$ and $\boldsymbol{K}^{\mathrm{dn}}(n, n+1)=0_{M, M}$. In the same way we obtain

$$
\begin{aligned}
& \boldsymbol{L}^{\mathrm{up}}(n, j)=-2 i h \sum_{\tau=0}^{[(n-j-1) / 2]}(-1)^{\tau} \boldsymbol{u}_{j} \boldsymbol{L}^{\mathrm{dn}}(j, 2 j+2 \tau-n+1), \\
& \boldsymbol{L}^{\mathrm{dn}}(n, j)=2 i h \sum_{\tau=0}^{[(n-j-1) / 2]}(-1)^{n-j-\tau-1} \boldsymbol{w}_{j} \boldsymbol{L}^{\mathrm{up}}(j, 2 j+2 \tau-n+1), \\
& \overline{\boldsymbol{L}}^{\mathrm{up}}(n, j)=-2 i h \sum_{\tau=0}^{[(n-j-1) / 2]}(-1)^{n-j-\tau-1} \boldsymbol{u}_{j} \overline{\boldsymbol{L}}^{\mathrm{dn}}(j, 2 j+2 \tau-n+1), \\
& \overline{\boldsymbol{L}}^{\mathrm{dn}}(n, j)=2 i h \sum_{\tau=0}^{[(n-j-1) / 2]}(-1)^{\tau} \boldsymbol{w}_{j} \overline{\boldsymbol{L}}^{\mathrm{up}}(j, 2 j+2 \tau-n+1),
\end{aligned}
$$

where $j \leq n-1$. Using (2.9c) and (2.9d) we get

$$
\boldsymbol{L}^{\mathrm{dn}}(n, n-1)=2 i h \boldsymbol{w}_{n-1}, \quad \overline{\boldsymbol{L}}^{\mathrm{up}}(n, n-1)=-2 i h \boldsymbol{u}_{n-1},
$$

as well as $\overline{\boldsymbol{L}}^{\mathrm{up}}(n, n-1)=0_{N, N}$ and $\boldsymbol{L}^{\mathrm{dn}}(n, n-1)=0_{M, M}$. Putting

$$
\left\|\boldsymbol{K}^{\bullet}(n, \cdot)\right\|_{1}=\sum_{j=n}^{\infty}\left\|\boldsymbol{K}^{\bullet}(n, j)\right\|, \quad\left\|\boldsymbol{L}^{\bullet}(n, \cdot)\right\|_{1}=\sum_{j=-\infty}^{n}\left\|\boldsymbol{L}^{\bullet}(n, j)\right\|,
$$

where $\boldsymbol{K}^{\bullet} \in\left\{\boldsymbol{K}^{\text {up }}, \boldsymbol{K}^{\text {dn }}, \overline{\boldsymbol{K}}^{\text {up }}, \overline{\boldsymbol{K}}^{\mathrm{dn}}\right\}$ and $\boldsymbol{L}^{\bullet} \in\left\{\overline{\boldsymbol{L}}^{\text {up }}, \overline{\boldsymbol{L}}^{\mathrm{dn}}, \boldsymbol{L}^{\mathrm{up}}, \boldsymbol{L}^{\mathrm{dn}}\right\}$, we get the estimates

$$
\begin{aligned}
& \left\|\overline{\boldsymbol{K}}^{\mathrm{up}}(n, \cdot)\right\|_{1} \leq 1+2 h \sum_{j=n+1}^{\infty}\left\|\boldsymbol{u}_{j}\right\|\left\|\overline{\boldsymbol{K}}^{\mathrm{dn}}(j, \cdot)\right\|_{1}, \\
& \left\|\overline{\boldsymbol{K}}^{\mathrm{dn}}(n, \cdot)\right\|_{1} \leq 2 h \sum_{j=n+1}^{\infty}\left\|\boldsymbol{w}_{j}\right\|\left\|\overline{\boldsymbol{K}}^{\mathrm{up}}(j, \cdot)\right\|_{1} .
\end{aligned}
$$

Using Proposition A.1 we obtain the estimates

$$
\left\|\overline{\boldsymbol{K}}^{\mathrm{up}}(n, \cdot)\right\|_{1} \leq \exp \left(4 h^{2} U_{n} W_{n}\right), \quad\left\|\overline{\boldsymbol{K}}^{\mathrm{dn}}(n, \cdot)\right\|_{1} \leq 2 h U_{n} \exp \left(4 h^{2} U_{n} W_{n}\right),
$$

where $U_{n}=\sum_{j=n+1}^{\infty}\left\|\boldsymbol{u}_{j}\right\|$ and $W_{n}=\sum_{s=n+1}^{\infty}\left\|\boldsymbol{w}_{s}\right\|$. Similar estimates can be derived for the other quantities.

Equations (2.2a), (2.2b) and (2.4a), (2.4b) imply that

$$
\begin{aligned}
& \left(\overline{\boldsymbol{M}}_{n}(z) \quad \boldsymbol{M}_{n}(z)\right)=J\left(\overline{\boldsymbol{M}}_{n}(-z) \quad \boldsymbol{M}_{n}(-z)\right) J, \\
& \left(N_{n}(z) \quad \bar{N}_{n}(z)\right)=J\left(N_{n}(-z) \quad \bar{N}_{n}(-z)\right) J .
\end{aligned}
$$


Thus $\overline{\boldsymbol{K}}^{\text {up }}(n, m), \boldsymbol{K}^{\mathrm{dn}}(n, m), \boldsymbol{L}^{\mathrm{up}}(n, m)$, and $\overline{\boldsymbol{L}}^{\mathrm{dn}}(n, m)$ vanish if $m-n$ is odd, and $\overline{\boldsymbol{K}}^{\mathrm{dn}}(n, m), \boldsymbol{K}^{\mathrm{up}}(n, m), \boldsymbol{L}^{\mathrm{dn}}(n, m)$, and $\overline{\boldsymbol{L}}^{\text {up }}(n, m)$ vanish if $m-n$ is even.

Proposition 2.3 (Uniform boundedness of Jost solutions) Let either potential $\left\{\boldsymbol{u}_{n}\right\}_{n=-\infty}^{\infty}$ and $\left\{\boldsymbol{w}_{n}\right\}_{n=-\infty}^{\infty}$ satisfy the $\ell^{1}$-condition (1.3). Then for $\pm i \neq z \in \mathbb{T}$ the Jost solutions $\psi_{n}(z)$, $\bar{\psi}_{n}(z), \phi_{n}(z)$, and $\bar{\phi}_{n}(z)$ of the discrete matrix Zakharov-Shabat system (1.5) are uniformly bounded in $n \in \mathbb{Z}$.

Proof Since Jost solutions and corresponding Faddeev functions differ by a unimodular factor, it suffices to prove Proposition 2.3 for Faddeev functions. We shall therefore focus on the solutions to the system (2.7a)-(2.7b). Putting $C(z)=\left|2 z /\left(z^{2}+1\right)\right|$ for $0 \neq i \in \mathbb{T}$, we arrive at the following estimates:

$$
\begin{aligned}
& \left\|\overline{\boldsymbol{N}}_{n}^{\mathrm{up}}(z)\right\| \leq 1+2 h C(z) \sum_{j=n+1}^{\infty}\left\|\boldsymbol{u}_{j}\right\|\left\|\overline{\boldsymbol{N}}_{n}^{\mathrm{dn}}(z)\right\|, \\
& \left\|\overline{\boldsymbol{N}}_{n}^{\mathrm{dn}}(z)\right\| \leq 2 h C(z) \sum_{j=n+1}^{\infty}\left\|\boldsymbol{w}_{j}\right\|\left\|\overline{\boldsymbol{N}}_{n}^{\mathrm{up}}(z)\right\| .
\end{aligned}
$$

Summing these two inequalities and applying Proposition A.1 we get

$$
\left\|\overline{\boldsymbol{N}}_{n}^{\mathrm{up}}(z)\right\|+\left\|\overline{\boldsymbol{N}}_{n}^{\mathrm{dn}}(z)\right\| \leq \exp \left(h C(z) \sum_{j=n+1}^{\infty} \max \left(\left\|\boldsymbol{u}_{j}\right\|,\left\|\boldsymbol{w}_{j}\right\|\right)\right),
$$

which completes the proof.

For $z \in \mathbb{T}$ we use the bounds

$$
\left|\sum_{\tau=0}^{j-n-1}(-1)^{\tau} z^{2 \tau}\right| \leq j-n, \quad\left|\sum_{\tau=0}^{j-n-1}(-1)^{\tau} z^{2(j-n-\tau-1)}\right| \leq j-n,
$$

plus the preceding argument to arrive at the estimate

$$
\left\|\overline{\boldsymbol{N}}_{n}^{\mathrm{up}}(z)\right\|+\left\|\overline{\boldsymbol{N}}_{n}^{\mathrm{dn}}(z)\right\| \leq \exp \left(2 h \sum_{j=n+1}^{\infty}(j-n) \max \left(\left\|\boldsymbol{u}_{j}\right\|,\left\|\boldsymbol{w}_{j}\right\|\right)\right),
$$

valid under the Faddeev condition (2.3). Thus for $z= \pm i$ the uniform boundedness result only holds in exceptional circumstances.

\section{Transition Coefficients}

In this section we prove, for $0 \neq z \in \mathbb{T}$, the existence of so-called transition matrices $\boldsymbol{T}(z)$ and $\overline{\boldsymbol{T}}(z)$ such that

$$
\begin{aligned}
& \left(\phi_{n}(z) \quad \bar{\phi}_{n}(z)\right)=\left(\bar{\psi}_{n}(z) \quad \psi_{n}(z)\right) \boldsymbol{T}(z), \\
& \left(\bar{\psi}_{n}(z) \quad \psi_{n}(z)\right)=\left(\phi_{n}(z) \quad \bar{\phi}_{n}(z)\right) \overline{\boldsymbol{T}}(z),
\end{aligned}
$$


where

$$
\boldsymbol{T}(z)=\left(\begin{array}{ll}
\boldsymbol{a}(z) & \overline{\boldsymbol{b}}(z) \\
\boldsymbol{b}(z) & \overline{\boldsymbol{a}}(z)
\end{array}\right), \quad \overline{\boldsymbol{T}}(z)=\left(\begin{array}{ll}
\overline{\boldsymbol{c}}(z) & \boldsymbol{d}(z) \\
\overline{\boldsymbol{d}}(z) & \boldsymbol{c}(z)
\end{array}\right)
$$

Here $\boldsymbol{a}(z), \boldsymbol{b}(z), \overline{\boldsymbol{a}}(z), \overline{\boldsymbol{b}}(z), \overline{\boldsymbol{c}}(z), \overline{\boldsymbol{d}}(z), \boldsymbol{c}(z)$, and $\boldsymbol{d}(z)$ are called transition coefficients.

Using (2.4a), (2.4b), we write (3.1a), (3.1b) in the form

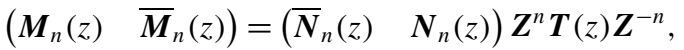

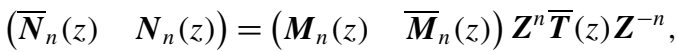

Because the left-hand sides of (3.2a) and (3.2b) tend to $I_{N+M}$ as $n \rightarrow-\infty$ and $n \rightarrow+\infty$, respectively, the transition matrices $\boldsymbol{T}(z)$ and $\overline{\boldsymbol{T}}(z)$ are each other's inverses whenever $\pm i \neq$ $z \in \mathbb{T}$.

Using Proposition 2.3 and letting $n \rightarrow \pm \infty$ in each block of (3.2a), (3.2b), we immediately have the following justification of the existence of the transition coefficients.

Proposition 3.1 (Expressing transition coefficients) Let the potentials $\left\{\boldsymbol{u}_{n}\right\}_{n=-\infty}^{\infty}$ and $\left\{\boldsymbol{w}_{n}\right\}_{n=-\infty}^{\infty}$ satisfy the Faddeev condition (2.3). Then for $\pm i \neq z \in \mathbb{T}$ we have

$$
\begin{aligned}
& \boldsymbol{a}(z)=I_{N}-\frac{2 i h z}{z^{2}+1} \sum_{j=-\infty}^{\infty} \boldsymbol{u}_{j} \boldsymbol{M}_{j}^{d n}(z), \\
& \overline{\boldsymbol{a}}(z)=I_{M}+\frac{2 i h z}{z^{2}+1} \sum_{j=-\infty}^{\infty} \boldsymbol{w}_{j} \overline{\boldsymbol{M}}_{j}^{u p}(z), \\
& \boldsymbol{b}(z)=\frac{2 i h z}{z^{2}+1} \sum_{j=-\infty}^{\infty} z^{2 j} \boldsymbol{w}_{j} \boldsymbol{M}_{j}^{u p}(z), \\
& \overline{\boldsymbol{b}}(z)=-\frac{2 i h z}{z^{2}+1} \sum_{j=-\infty}^{\infty} z^{-2 j} \boldsymbol{u}_{j} \overline{\boldsymbol{M}}_{j}^{d n}(z), \\
& \overline{\boldsymbol{c}}(z)=I_{N}-\frac{2 i h z}{z^{2}+1} \sum_{j=-\infty}^{\infty} \boldsymbol{u}_{j} \overline{\boldsymbol{N}}_{j}^{d n}(z), \\
& \boldsymbol{c}(z)=I_{M}+\frac{2 i h z}{z^{2}+1} \sum_{j=-\infty}^{\infty} \boldsymbol{w}_{j} \boldsymbol{N}_{j}^{u p}(z), \\
& \overline{\boldsymbol{d}}(z)=\frac{2 i h z}{z^{2}+1} \sum_{j=-\infty}^{\infty} z^{2 j} \boldsymbol{w}_{j} \overline{\boldsymbol{N}}_{j}^{u p}(z), \\
& \boldsymbol{d}(z)=-\frac{2 i h z}{z^{2}+1} \sum_{j=-\infty}^{\infty} z^{-2 j} \boldsymbol{u}_{j} \boldsymbol{N}_{j}^{d n}(z),
\end{aligned}
$$

where the series are absolutely convergent uniformly in z belonging to any compact subset of $\mathbb{T} \backslash\{ \pm i\}$.

Using the invertibility of the transition matrices, we easily obtain 
Proposition 3.2 For $\pm i \neq \mathbb{T}$, the $N \times N$ matrix $\boldsymbol{a}(z)$ is invertible iff the $M \times M$ matrix $c(z)$ is invertible, and the $M \times M$ matrix $\overline{\boldsymbol{a}}(z)$ is invertible iff the $N \times N$ matrix $\overline{\boldsymbol{c}}(z)$ is invertible.

Proof Indeed, if $\overline{\boldsymbol{a}}$ is invertible, then $\overline{\boldsymbol{d}}=-\overline{\boldsymbol{a}}^{-1} \boldsymbol{b} \overline{\boldsymbol{c}}$ and $\left(\boldsymbol{a}-\overline{\boldsymbol{b}} \overline{\boldsymbol{a}}^{-1} \boldsymbol{b}\right) \overline{\boldsymbol{c}}=I_{N}$. If $\overline{\boldsymbol{c}}$ is invertible, then $\overline{\boldsymbol{b}}=-\overline{\boldsymbol{c}}^{-1} \boldsymbol{d} \overline{\boldsymbol{a}}$ and $\left(\boldsymbol{c}-\overline{\boldsymbol{d}} \overline{\boldsymbol{c}}^{-1} \boldsymbol{d}\right) \overline{\boldsymbol{a}}=I_{M}$. Also, if $\boldsymbol{a}$ is invertible, then $\boldsymbol{d}=-\boldsymbol{a}^{-1} \overline{\boldsymbol{b}} \boldsymbol{c}$ and $\left(\overline{\boldsymbol{a}}-\boldsymbol{b} \boldsymbol{a}^{-1} \overline{\boldsymbol{b}}\right) \boldsymbol{c}=I_{M}$. If $\boldsymbol{c}$ is invertible, then $\boldsymbol{b}=-\boldsymbol{c}^{-1} \overline{\boldsymbol{d}} \boldsymbol{a}$ and $\left(\overline{\boldsymbol{c}}-\boldsymbol{d} \boldsymbol{c}^{-1} \overline{\boldsymbol{d}}\right) \boldsymbol{a}=I_{N}$.

An $(N+M) \times(N+M)$ matrix $\boldsymbol{E}$ is called $J$-unitary if

$$
\boldsymbol{E}^{\dagger} J \boldsymbol{E}=J
$$

It is easily verified that the $J$-unitary matrices form a group which is invariant under the operation $\boldsymbol{E} \mapsto \boldsymbol{E}^{\dagger}$.

Proposition 3.3 (Wronskian relation) In the focusing case the transition matrices $\boldsymbol{T}(z)$ and $\overline{\boldsymbol{T}}(z)$ are unitary whenever $z \in \mathbb{T} \backslash\{ \pm i\}$. In the defocussing case the transition matrices $\boldsymbol{T}(z)$ and $\overline{\boldsymbol{T}}(z)$ are $J$-unitary whenever $z \in \mathbb{T} \backslash\{ \pm i\}$.

Proof For $z \in \mathbb{T}$, let $\boldsymbol{v}_{n}(z)$ and $\tilde{\boldsymbol{v}}_{n}(z)$ be square matrix solutions of (1.5). Then, in the focusing case,

$$
\begin{aligned}
J \tilde{\boldsymbol{v}}_{n+1}(z)^{\dagger} J-J \tilde{\boldsymbol{v}}_{n-1}(z)^{\dagger} J & =-2 i h\left[J \tilde{\boldsymbol{v}}_{n}(z)^{\dagger} J\right] J\left(\begin{array}{cc}
\lambda I_{N} & -\boldsymbol{u}_{n} \\
-\boldsymbol{w}_{n} & \lambda I_{M}
\end{array}\right) \\
& =-2 i h\left[J \tilde{\boldsymbol{v}}_{n}(z)^{\dagger} J\right]\left(\begin{array}{cc}
\lambda I_{N} & \boldsymbol{u}_{n} \\
\boldsymbol{w}_{n} & \lambda I_{M}
\end{array}\right) J
\end{aligned}
$$

where we have used that $\boldsymbol{w}_{n}=-\boldsymbol{u}_{n}^{\dagger}$. Thus, we get the Wronskian relation

$$
\left[J \tilde{\boldsymbol{v}}_{n+1}(z)^{\dagger} J-J \tilde{\boldsymbol{v}}_{n-1}(z)^{\dagger} J\right] J \boldsymbol{v}_{n}(z)=-J \tilde{\boldsymbol{v}}_{n}(z)^{\dagger}\left[\boldsymbol{v}_{n+1}(z)-\boldsymbol{v}_{n-1}(z)\right] .
$$

Hence the expression

$$
\tilde{\boldsymbol{v}}_{n+1}(z)^{\dagger} \boldsymbol{v}_{n}(z)+\tilde{\boldsymbol{v}}_{n}(z)^{\dagger} \boldsymbol{v}_{n+1}(z)
$$

does not depend on $n \in \mathbb{Z}$ whenever $z \in \mathbb{T}$. Equating the asymptotic behaviors of this expression as $n \rightarrow \pm \infty$, we obtain for $\boldsymbol{v}_{n}=\tilde{\boldsymbol{v}}_{n}=\left(\bar{\psi}_{n} \psi_{n}\right)$

$$
\overline{\boldsymbol{T}}(z)^{\dagger}\left(\boldsymbol{Z}+\boldsymbol{Z}^{-1}\right) \overline{\boldsymbol{T}}(z)=\boldsymbol{Z}+\boldsymbol{Z}^{-1},
$$

where $\boldsymbol{Z}=\boldsymbol{Z}^{\dagger}=\operatorname{diag}\left(z I_{N}, z^{-1} I_{M}\right)$. For $\boldsymbol{v}_{n}=\tilde{\boldsymbol{v}}_{n}=\left(\phi_{n} \bar{\phi}_{n}\right)$ we get

$$
\boldsymbol{T}(z)^{\dagger}\left(\boldsymbol{Z}+\boldsymbol{Z}^{-1}\right) \boldsymbol{T}(z)=\boldsymbol{Z}+\boldsymbol{Z}^{-1} .
$$

For $\boldsymbol{v}_{n}=\left(\bar{\psi}_{n} \psi_{n}\right)$ and $\tilde{\boldsymbol{v}}_{n}=\left(\phi_{n} \bar{\phi}_{n}\right)$ we obtain

$$
\overline{\boldsymbol{T}}(z)^{\dagger}\left(\boldsymbol{Z}+\boldsymbol{Z}^{-1}\right)=\left(\boldsymbol{Z}+\boldsymbol{Z}^{-1}\right) \boldsymbol{T}(z) .
$$

However, $\boldsymbol{Z}+\boldsymbol{Z}^{-1}=\left(z+z^{-1}\right) I_{N+M}$, a nonsingular multiple of the identity matrix unless $z= \pm i$. Thus for $z \in \mathbb{T} \backslash\{ \pm i\}$ we get

$$
\overline{\boldsymbol{T}}(z)^{\dagger} \overline{\boldsymbol{T}}(z)=I_{N+M}, \quad \boldsymbol{T}(z)^{\dagger} \boldsymbol{T}(z)=I_{N+M}, \quad \overline{\boldsymbol{T}}(z)^{\dagger}=\boldsymbol{T}(z),
$$


as claimed.

In the defocussing case, we prove instead that

$$
\tilde{\boldsymbol{v}}_{n+1}(z)^{\dagger} \boldsymbol{J} \boldsymbol{v}_{n}(z)+\tilde{\boldsymbol{v}}_{n}(z)^{\dagger} J \boldsymbol{v}_{n+1}(z)
$$

does not depend on $n \in \mathbb{Z}$. In this case we get

$$
\overline{\boldsymbol{T}}(z)^{\dagger} J \overline{\boldsymbol{T}}(z)=J, \quad \boldsymbol{T}(z)^{\dagger} J \boldsymbol{T}(z)=J, \quad \overline{\boldsymbol{T}}(z)^{\dagger}=J \boldsymbol{T}(z) J,
$$

which completes the proof.

Let us return to parity symmetry. Using (2.2a), (2.2b) and (3.1a), (3.1b) it is easily shown that the transition matrices satisfy the parity relations

$$
\boldsymbol{T}(z)=J \boldsymbol{T}(-z) J, \quad \bar{T}(z)=J \overline{\boldsymbol{T}}(-z) J .
$$

\section{Reflection and Transmission Coefficients}

In this section we define the reflection and transmission coefficients and derive the Marchenko equations if there are no spectral singularities. By a spectral singularity at $z \in \mathbb{T} \backslash\{ \pm i\}$ we mean a complex number $\pm i \neq z \in \mathbb{T}$ for which at least one of the matrices $\boldsymbol{a}(z)$ [or, equivalently, $\boldsymbol{c}(z)$ ] and $\overline{\boldsymbol{a}}(z)$ [or, equivalently, $\overline{\boldsymbol{d}}(z)$ ] is singular. Parity symmetry implies that $z \in \mathbb{T} \backslash\{ \pm i\}$ is a spectral singularity whenever $-z$ is.

Assume there are no spectral singularities. Then (3.1a), (3.1b) can be written as the following Riemann-Hilbert problems:

$$
\begin{aligned}
& \bar{\psi}_{n}(z)=\left[\phi_{n}(z)-\psi_{n}(z) \boldsymbol{b}(z)\right] \boldsymbol{a}(z)^{-1}, \\
& \psi_{n}(z)=\left[\bar{\phi}_{n}(z)-\bar{\psi}_{n}(z) \overline{\boldsymbol{b}}(z)\right] \overline{\boldsymbol{a}}(z)^{-1}, \\
& \phi_{n}(z)=\left[\bar{\psi}_{n}(z)-\bar{\phi}_{n}(z) \overline{\boldsymbol{d}}(z)\right] \overline{\boldsymbol{c}}(z)^{-1}, \\
& \bar{\phi}_{n}(z)=\left[\psi_{n}(z)-\phi_{n}(z) \boldsymbol{d}(z)\right] \boldsymbol{c}(z)^{-1} .
\end{aligned}
$$

Defining the transmission coefficients $\boldsymbol{t}_{r}(z), \overline{\boldsymbol{t}}_{r}(z), \overline{\boldsymbol{t}}_{l}(z)$, and $\boldsymbol{t}_{l}(z)$ and the reflection coefficients $\rho(z), \bar{\rho}(z), \bar{\ell}(z)$, and $\ell(z)$ by

$$
\begin{aligned}
& \boldsymbol{t}_{r}(z)=\boldsymbol{a}(z)^{-1}=\overline{\boldsymbol{c}}(z)-\boldsymbol{d}(z) \boldsymbol{c}(z)^{-1} \overline{\boldsymbol{d}}(z), \\
& \overline{\boldsymbol{t}}_{r}(z)=\overline{\boldsymbol{a}}(z)^{-1}=\boldsymbol{c}(z)-\overline{\boldsymbol{d}}(z) \overline{\boldsymbol{c}}(z)^{-1} \boldsymbol{d}(z), \\
& \boldsymbol{\rho}(z)=\boldsymbol{b}(z) \boldsymbol{a}(z)^{-1}=-\boldsymbol{c}(z)^{-1} \overline{\boldsymbol{d}}(z), \\
& \overline{\boldsymbol{\rho}}(z)=\overline{\boldsymbol{b}}(z) \overline{\boldsymbol{a}}(z)^{-1}=-\overline{\boldsymbol{c}}(z)^{-1} \boldsymbol{d}(z), \\
& \overline{\boldsymbol{t}}_{l}(z)=\overline{\boldsymbol{c}}(z)^{-1}=\boldsymbol{a}(z)-\overline{\boldsymbol{b}}(z) \overline{\boldsymbol{a}}(z)^{-1} \boldsymbol{b}(z), \\
& \boldsymbol{t}_{l}(z)=\boldsymbol{c}(z)^{-1}=\overline{\boldsymbol{a}}(z)-\boldsymbol{b}(z) \boldsymbol{a}(z)^{-1} \overline{\boldsymbol{b}}(z), \\
& \overline{\boldsymbol{\ell}}(z)=\overline{\boldsymbol{d}}(z) \overline{\boldsymbol{c}}(z)^{-1}=-\overline{\boldsymbol{a}}(z)^{-1} \boldsymbol{b}(z), \\
& \boldsymbol{\ell}(z)=\boldsymbol{d}(z) \boldsymbol{c}(z)^{-1}=-\boldsymbol{a}(z)^{-1} \overline{\boldsymbol{b}}(z),
\end{aligned}
$$


we can write the Riemann-Hilbert problems in the following way:

$$
\begin{aligned}
\bar{\psi}_{n}(z) & =\phi_{n}(z) \boldsymbol{t}_{r}(z)-\psi_{n}(z) \rho(z), \\
\psi_{n}(z) & =\bar{\phi}_{n}(z) \overline{\boldsymbol{t}}_{r}(z)-\bar{\psi}_{n}(z) \overline{\boldsymbol{\rho}}(z), \\
\phi_{n}(z) & =\bar{\psi}_{n}(z) \overline{\boldsymbol{t}}_{l}(z)-\bar{\phi}_{n}(z) \overline{\boldsymbol{\ell}}(z), \\
\bar{\phi}_{n}(z) & =\psi_{n}(z) \boldsymbol{t}_{l}(z)-\phi_{n}(z) \boldsymbol{\ell}(z) .
\end{aligned}
$$

In other words,

$$
\begin{aligned}
& \left(\bar{\psi}_{n}(z) \quad \bar{\phi}_{n}(z)\right)=\left(\phi_{n}(z) \quad \psi_{n}(z)\right) J \boldsymbol{S}(z) J, \\
& \left(\phi_{n}(z) \quad \psi_{n}(z)\right)=\left(\bar{\psi}_{n}(z) \quad \bar{\phi}_{n}(z)\right) J \overline{\boldsymbol{S}}(z) J,
\end{aligned}
$$

where the matrices given by

$$
\boldsymbol{S}(z)=\left(\begin{array}{cc}
\boldsymbol{t}_{r}(z) & \ell(z) \\
\rho(z) & \boldsymbol{t}_{l}(z)
\end{array}\right), \quad \overline{\boldsymbol{S}}(z)=\left(\begin{array}{cc}
\overline{\boldsymbol{t}}_{l}(z) & \overline{\boldsymbol{\rho}}(z) \\
\bar{\ell}(z) & \overline{\boldsymbol{t}}_{r}(z)
\end{array}\right),
$$

are called scattering matrices. The alternative representations of the reflection coefficients in (4.1a)-(4.1h) follow from the fact that the transition matrix $\boldsymbol{T}(z)$ in (3.1a) is the inverse of the transition matrix $\overline{\boldsymbol{T}}(z)$ in (3.1b). Equations (4.3a), (4.3b) and (2.2a), (2.2b) imply the parity symmetry relations

$$
\boldsymbol{S}(z)=J \boldsymbol{S}(-z) J, \quad \overline{\boldsymbol{S}}(z)=J \overline{\boldsymbol{S}}(-z) J,
$$

which implies that the transmission coefficients are even functions of $z$ and the reflection coefficients are odd functions of $z$. Finally, in the focusing case $\boldsymbol{S}(z)$ and $\overline{\boldsymbol{S}}(z)$ are $J$-unitary matrices. In particular, for $\pm i \neq z \in \mathbb{T}$ we have

$$
\boldsymbol{t}_{l}(z)=\overline{\boldsymbol{t}}_{r}(z)^{\dagger}, \quad \overline{\boldsymbol{t}}_{l}(z)=\boldsymbol{t}_{r}(z)^{\dagger}, \quad \bar{\ell}(z)=-\ell(z)^{\dagger}, \quad \overline{\boldsymbol{\rho}}(z)=-\boldsymbol{\rho}(z)^{\dagger} .
$$

In the defocussing case the scattering matrices are unitary.

Using the Faddeev condition (2.3), we easily prove that, when multiplied by $\left(1+z^{2}\right) / 2 i z$, the matrix functions $I_{N}-\boldsymbol{a}(z), \overline{\boldsymbol{a}}(z)-I_{M}, \boldsymbol{b}(z), \overline{\boldsymbol{b}}(z), \overline{\boldsymbol{c}}(z)-I_{N}, I_{M}-\boldsymbol{c}(z), \overline{\boldsymbol{d}}(z)$, and $\boldsymbol{d}(z)$ belong to $\mathcal{W}_{-}^{N, N}, \mathcal{W}_{+}^{M, M}, \mathcal{W}^{M, N}, \mathcal{W}^{N, M}, \mathcal{W}_{+}^{N, N}, \mathcal{W}_{-}^{M, M}, \mathcal{W}^{N, M}$, and $\mathcal{W}^{M, N}$, respectively. For instance, in the case of (3.3a) we have

$$
\frac{1+z^{2}}{2 i z}\left[I_{N}-\boldsymbol{a}(z)\right]=h \sum_{s=0}^{\infty} z^{-s} \sum_{k=-\infty}^{\infty} \boldsymbol{u}_{k} \boldsymbol{L}^{\mathrm{dn}}(k, k-s),
$$

where, for $U_{-\infty}=\sum_{k=-\infty}^{\infty}\left\|\boldsymbol{u}_{k}\right\|$ and $W_{-\infty}=\sum_{k=-\infty}^{\infty}\left\|\boldsymbol{w}_{k}\right\|$,

$$
\sum_{s=0}^{\infty}\left\|\sum_{k=-\infty}^{\infty} \boldsymbol{u}_{k} \boldsymbol{L}^{\mathrm{dn}}(k, k-s)\right\| \leq 2 h^{2} U_{-\infty} W_{-\infty} \exp \left(4 h^{2} U_{-\infty} W_{-\infty}\right)<+\infty .
$$

Let us now write the reflection coefficients as follows:

$$
\boldsymbol{\rho}(z)=\sum_{s=-\infty}^{\infty} z^{s} \hat{\boldsymbol{\rho}}(s), \quad \overline{\boldsymbol{\rho}}(z)=\sum_{s=-\infty}^{\infty} z^{-s \hat{\bar{\rho}}}(s),
$$




$$
\bar{\ell}(z)=\sum_{s=-\infty}^{\infty} z^{s \hat{\bar{\ell}}}(s), \quad \ell(z)=\sum_{s=-\infty}^{\infty} z^{-s} \hat{\ell}(s) .
$$

Parity symmetry implies that $\hat{\rho}(s), \hat{\bar{\rho}}(s), \hat{\ell}(s)$, and $\hat{\bar{\ell}}(s)$ vanish if $s$ is even.

In analogy with the situation for the Schrödinger equation on the line $[8,13]$, we say that the generic case holds at $z= \pm i$ if the four matrices $\left(1+z^{2}\right) \boldsymbol{a}(z),\left(1+z^{2}\right) \overline{\boldsymbol{a}}(z),\left(1+z^{2}\right) \overline{\boldsymbol{c}}(z)$ and $\left(1+z^{2}\right) \boldsymbol{c}(z)$ have nonsingular matrices as their limits as $z \rightarrow \pm i$ along $\mathbb{T}$. In all other cases, we say that the exceptional case holds at $z= \pm i$. Because of parity symmetry, the generic or the exceptional case can only hold at both points $z= \pm i$ or not hold at both points $z= \pm i$. We therefore omit the clause "at $z= \pm i$ " when discussing these cases.

Proposition 4.1 (Scattering coefficients as Fourier sums) Let the potentials $\left\{\boldsymbol{u}_{n}\right\}_{n=-\infty}^{\infty}$ and $\left\{\boldsymbol{w}_{n}\right\}_{n=-\infty}^{\infty}$ satisfy the Faddeev condition (2.3). Assume there are no spectral singularities, and the generic case holds. Then the reflection and transmission coefficients belong to $\mathcal{W}^{p, q}$ for certain $p$ and $q$.

Proof Clearly $\left(1+z^{2}\right) / 2 i z$ belongs to $\mathcal{W}^{1,1}$ and hence $\boldsymbol{a}(z)-I_{N}, \overline{\boldsymbol{a}}(z)-I_{M}, \overline{\boldsymbol{c}}(z)-I_{N}$, and $\boldsymbol{c}(z)-I_{M}$ belong to some $\mathcal{W}^{p, q}$ when multiplied by $\left(1+z^{2}\right) / 2 i z$. Thus $\left[\left(1+z^{2}\right) / 2 i z\right] \boldsymbol{a}(z)$, $\left[\left(1+z^{2}\right) / 2 i z\right] \overline{\boldsymbol{a}}(z),\left[\left(1+z^{2}\right) / 2 i z\right] \overline{\boldsymbol{c}}(z)$, and $\left[\left(1+z^{2}\right) / 2 i z\right] \boldsymbol{c}(z)$ belong to $\mathcal{W}^{p, q}$ for certain $p, q$. Because we are in the generic case and there are no spectral singularities, these four matrices are nonsingular for each $z \in \mathbb{T}$. By Wiener's theorem [26], their respective inverses $2 i z \boldsymbol{t}_{r}(z) /\left(1+z^{2}\right), 2 i z \overline{\boldsymbol{t}}_{r}(z) /\left(1+z^{2}\right), 2 i z \overline{\boldsymbol{t}}_{l}(z) /\left(1+z^{2}\right)$, and $2 i z \boldsymbol{t}_{l}(z) /\left(1+z^{2}\right)$ belong to $\mathcal{W}^{p, q}$ for certain $p, q$. This is also the case for the transmission coefficients, since they arise by multiplying these matrix functions by $\left(1+z^{2}\right) / 2 i z$.

Analogously, under the Faddeev condition (2.3) the left-hand sides of (3.3c), (3.3d), (3.3g), and (3.3h) multiplied by $\left(z^{2}+1\right) / 2 i z$ belong to $\mathcal{W}^{p, q}$ for certain $p, q$. Since $2 i z \boldsymbol{t}_{r}(z) /\left(1+z^{2}\right), 2 i z \overline{\boldsymbol{t}}_{r}(z) /\left(1+z^{2}\right), 2 i z \overline{\boldsymbol{t}}_{l}(z) /\left(1+z^{2}\right)$, and $2 i z \boldsymbol{t}_{l}(z) /\left(1+z^{2}\right)$ also belong to $\mathcal{W}^{p, q}$ for certain $p, q$, their respective products, i.e., the reflection coefficients, belong to $\mathcal{W}^{p, q}$ for certain $p, q$.

\section{Marchenko Equations}

Throughout this section we assume that the reflection coefficients $\rho, \ell \in \mathcal{W}^{N, M}$ and $\bar{\rho}, \bar{\ell} \in$ $\mathcal{W}^{M, N}$. This occurs, for instance, if the generic case holds and there are no spectral singularities. We shall derive the Marchenko equations, first if the poles of the corresponding transmission coefficients are finite in number and simple and then more generally. We shall also apply parity symmetry to write the Marchenko equations in a form more suitable for deriving closed form solutions.

Use (2.4a), (2.4b) to write (4.2a)-(4.2d) in the form

$$
\begin{aligned}
\overline{\boldsymbol{N}}_{n}(z) & =\boldsymbol{M}_{n}(z) \boldsymbol{t}_{r}(z)-z^{-2 n} \boldsymbol{N}_{n}(z) \boldsymbol{\rho}(z), \\
\boldsymbol{N}_{n}(z) & =\overline{\boldsymbol{M}}_{n}(z) \overline{\boldsymbol{t}}_{r}(z)-z^{2 n} \overline{\boldsymbol{N}}_{n}(z) \overline{\boldsymbol{\rho}}(z), \\
\boldsymbol{M}_{n}(z) & =\overline{\boldsymbol{N}}_{n}(z) \overline{\boldsymbol{t}}_{l}(z)-z^{-2 n} \overline{\boldsymbol{M}}_{n}(z) \overline{\boldsymbol{\ell}}(z), \\
\overline{\boldsymbol{M}}_{n}(z) & =\boldsymbol{N}_{n}(z) \boldsymbol{t}_{l}(z)-z^{2 n} \boldsymbol{M}_{n}(z) \boldsymbol{\ell}(z),
\end{aligned}
$$


respectively. Putting

$$
\begin{array}{ll}
\boldsymbol{t}_{r}(z)=\boldsymbol{t}_{r 0}(z)+\sum_{k} \frac{\boldsymbol{t}_{r k}}{z-\zeta_{k}}, & \overline{\boldsymbol{t}}_{r}(z)=\overline{\boldsymbol{t}}_{r 0}(z)+\sum_{k} \frac{\overline{\boldsymbol{t}}_{r k}}{z-\bar{\zeta}_{k}}, \\
\overline{\boldsymbol{t}}_{l}(z)=\overline{\boldsymbol{t}}_{l 0}(z)+\sum_{k} \frac{\overline{\boldsymbol{t}}_{l k}}{z-\bar{\zeta}_{k}}, & \boldsymbol{t}_{l}(z)=\boldsymbol{t}_{l 0}(z)+\sum_{k} \frac{\boldsymbol{t}_{l k}}{z-\zeta_{k}},
\end{array}
$$

where $\boldsymbol{t}_{r 0} \in \mathcal{W}_{-}^{N, N}, \overline{\boldsymbol{t}}_{r 0} \in \mathcal{W}_{+}^{M, M}, \overline{\boldsymbol{t}}_{l 0} \in \mathcal{W}_{+}^{N, N}$, and $\boldsymbol{t}_{l, 0} \in \mathcal{W}_{-}^{M, M}, \zeta_{k}$ are finitely many distinct numbers with $\left|\zeta_{k}\right|>1$, and $\bar{\zeta}_{k}$ are finitely many distinct numbers with $0<\left|\bar{\zeta}_{k}\right|<1$, we obtain

$$
\begin{aligned}
& \overline{\boldsymbol{N}}_{n}(z)=\left(\begin{array}{c}
I_{N} \\
0_{M, N}
\end{array}\right)+\sum_{k} \frac{\boldsymbol{M}_{n}\left(\zeta_{k}\right) \boldsymbol{t}_{r k}}{z-\zeta_{k}}-\Pi_{+}\left(z^{-2 n} \boldsymbol{N}_{n}(z) \boldsymbol{\rho}(z)\right), \\
& \boldsymbol{N}_{n}(z)=\left(\begin{array}{c}
0_{N, M} \\
I_{M}
\end{array}\right)+\sum_{k} \frac{\overline{\boldsymbol{M}}_{n}\left(\bar{\zeta}_{k}\right) \overline{\boldsymbol{t}}_{r k}}{z-\bar{\zeta}_{k}}-\Pi_{-}\left(z^{2 n} \overline{\boldsymbol{N}}_{n}(z) \overline{\boldsymbol{\rho}}(z)\right), \\
& \boldsymbol{M}_{n}(z)=\left(\begin{array}{c}
I_{N} \\
0_{M, N}
\end{array}\right)+\sum_{k} \frac{\overline{\boldsymbol{N}}_{n}\left(\bar{\zeta}_{k}\right) \overline{\boldsymbol{t}}_{l k}}{z-\bar{\zeta}_{k}}-\Pi_{-}\left(z^{-2 n} \overline{\boldsymbol{M}}_{n}(z) \overline{\boldsymbol{\ell}}(z)\right), \\
& \overline{\boldsymbol{M}}_{n}(z)=\left(\begin{array}{c}
0_{N, M} \\
I_{M}
\end{array}\right)+\sum_{k} \frac{\boldsymbol{N}_{n}\left(\zeta_{k}\right) \boldsymbol{t}_{l k}}{z-\zeta_{k}}-\Pi_{+}\left(z^{2 n} \boldsymbol{M}_{n}(z) \boldsymbol{\ell}(z)\right) .
\end{aligned}
$$

Here $\Pi_{+}$and $\Pi_{-}$are the natural projections of $\mathcal{W}^{p, q}$ onto $\mathcal{W}_{+}^{p, q}$ and $\mathcal{W}_{-}^{p, q}$, respectively.

Let us now introduce the so-called norming constants $\boldsymbol{C}_{r k}, \overline{\boldsymbol{C}}_{r k}, \overline{\boldsymbol{C}}_{l k}$, and $\boldsymbol{C}_{l k}$ such that

$$
\begin{array}{rlrl}
\boldsymbol{M}_{n}\left(\zeta_{k}\right) \boldsymbol{t}_{r k} & =\zeta_{k}^{-2 n} \boldsymbol{N}_{n}\left(\zeta_{k}\right) \boldsymbol{C}_{r k}, & \overline{\boldsymbol{M}}_{n}\left(\bar{\zeta}_{k}\right) \overline{\boldsymbol{t}}_{r k} & =-\bar{\zeta}_{k}^{2 n} \overline{\boldsymbol{N}}_{n}\left(\bar{\zeta}_{k}\right) \overline{\boldsymbol{C}}_{r k}, \\
\overline{\boldsymbol{N}}_{n}\left(\bar{\zeta}_{k}\right) \overline{\boldsymbol{t}}_{l k}=-\bar{\zeta}_{k}^{-2 n} \overline{\boldsymbol{M}}_{n}\left(\bar{\zeta}_{k}\right) \overline{\boldsymbol{C}}_{l k}, & \boldsymbol{N}_{n}\left(\zeta_{k}\right) \boldsymbol{t}_{l k}=\zeta_{k}^{2 n} \boldsymbol{M}_{n}\left(\zeta_{k}\right) \boldsymbol{C}_{l k} .
\end{array}
$$

Recalling that the transmission coefficients are even functions of $z$, their residues at the poles $\pm \zeta_{k}$ (or $\pm \bar{\zeta}_{k}$ ) add up to zero. Taking into account the parity properties of the Faddeev functions we see that the norming constants pertaining to the poles $\pm \zeta_{k}$ (or $\pm \bar{\zeta}_{k}$ ) coincide.

Substituting (5.3a), (5.3b) into (5.2a), (5.2b) and using (2.8a)-(2.8d), we collect the coefficients in the four power series in $z$ or $z^{-1}$ and obtain the Marchenko equations

$$
\begin{gathered}
\overline{\boldsymbol{K}}(n, m)+\sum_{j=n}^{\infty} \boldsymbol{K}(n, j) \boldsymbol{F}_{r}(j+m)=\left(\begin{array}{c}
I_{N} \\
0_{M, N}
\end{array}\right) \delta_{n, m}, \\
\boldsymbol{K}(n, m)+\sum_{j=n}^{\infty} \overline{\boldsymbol{K}}(n, j) \overline{\boldsymbol{F}}_{r}(j+m)=\left(\begin{array}{c}
0_{N, M} \\
I_{M}
\end{array}\right) \delta_{n, m},
\end{gathered}
$$

for $m \geq n$, and

$$
\begin{aligned}
& \boldsymbol{L}(n, m)+\sum_{j=-\infty}^{n} \overline{\boldsymbol{L}}(n, j) \overline{\boldsymbol{F}}_{l}(j+m)=\left(\begin{array}{c}
I_{N} \\
0_{M, N}
\end{array}\right) \delta_{n, m}, \\
& \overline{\boldsymbol{L}}(n, m)+\sum_{j=-\infty}^{n} \boldsymbol{L}(n, j) \boldsymbol{F}_{l}(j+m)=\left(\begin{array}{c}
0_{N, M} \\
I_{M}
\end{array}\right) \delta_{n, m},
\end{aligned}
$$


where $m \leq n$. Here $\delta_{n, m}$ is the Kronecker delta. The Marchenko kernels are given by

$$
\begin{array}{lll}
\boldsymbol{F}_{r}(s)=\hat{\boldsymbol{\rho}}(s)+\sum_{k} \zeta_{k}^{-(s+1)} \boldsymbol{C}_{r k}, & \overline{\boldsymbol{F}}_{r}(s)=\hat{\overline{\boldsymbol{\rho}}}(s)+\sum_{k} \bar{\zeta}_{k}^{s-1} \overline{\boldsymbol{C}}_{r k}, \\
\overline{\boldsymbol{F}}_{l}(s)=\hat{\overline{\boldsymbol{\ell}}}(s)+\sum_{k} \bar{\zeta}_{k}^{-(s+1)} \overline{\boldsymbol{C}}_{l k}, & \boldsymbol{F}_{l}(s)=\hat{\boldsymbol{\ell}}(s)+\sum_{k} \zeta_{k}^{s-1} \boldsymbol{C}_{l k} .
\end{array}
$$

Given the parity properties of the norming constants explained above, we see that $\boldsymbol{F}_{r}(s)$, $\overline{\boldsymbol{F}}_{r}(s), \overline{\boldsymbol{F}}_{l}(s)$, and $\boldsymbol{F}_{l}(s)$ vanish if $s$ is even. Using (2.9a)-(2.9d) we write (5.4a)-(5.4d) as follows:

$$
\begin{aligned}
& \overline{\boldsymbol{K}}(n, m)+\left(\begin{array}{c}
0_{N, M} \\
I_{M}
\end{array}\right) \boldsymbol{F}_{r}(n+m)+\sum_{j=n+1}^{\infty} \boldsymbol{K}(n, j) \boldsymbol{F}_{r}(j+m)=0, \\
& \boldsymbol{K}(n, m)+\left(\begin{array}{c}
I_{N} \\
0_{M, N}
\end{array}\right) \overline{\boldsymbol{F}}_{r}(n+m)+\sum_{j=n+1}^{\infty} \overline{\boldsymbol{K}}(n, j) \overline{\boldsymbol{F}}_{r}(j+m)=0,
\end{aligned}
$$

for $m \geq n+1$, and

$$
\begin{aligned}
& \boldsymbol{L}(n, m)+\left(\begin{array}{c}
0_{N, M} \\
I_{M}
\end{array}\right) \overline{\boldsymbol{F}}_{l}(n+m)+\sum_{j=-\infty}^{n-1} \overline{\boldsymbol{L}}(n, j) \overline{\boldsymbol{F}}_{l}(j+m)=0, \\
& \overline{\boldsymbol{L}}(n, m)+\left(\begin{array}{c}
I_{N} \\
0_{M, N}
\end{array}\right) \boldsymbol{F}_{l}(n+m)+\sum_{j=-\infty}^{n-1} \boldsymbol{L}(n, j) \boldsymbol{F}_{l}(j+m)=0,
\end{aligned}
$$

where $m \leq n-1$. Here the zeros in the right-hand sides are zero matrices with $N+M$ rows and either $N$ or $M$ columns.

To generalize the Marchenko equations (5.6a)-(5.6d) to the case where the transmission coefficients have finitely many, not necessarily simple, poles, we write the Marchenko kernels in the form

$$
\begin{array}{lll}
\boldsymbol{F}_{r}(s)=\hat{\boldsymbol{\rho}}(s)+C_{r} A_{r}^{-(s+1)} B_{r}, & \overline{\boldsymbol{F}}_{r}(s)=\hat{\overline{\boldsymbol{\rho}}}(s)+\bar{C}_{r} \bar{A}_{r}^{s-1} \bar{B}_{r}, \\
\overline{\boldsymbol{F}}_{l}(s)=\hat{\overline{\boldsymbol{\ell}}}(s)+\bar{C}_{l} \bar{A}_{l}^{-(s+1)} \bar{B}_{l}, & \boldsymbol{F}_{l}(s)=\hat{\boldsymbol{\ell}}(s)+C_{l} A_{l}^{s-1} B_{l},
\end{array}
$$

by introducing the four matrix triplets, $\left(A_{r}, B_{r}, C_{r}\right),\left(\bar{A}_{r}, \bar{B}_{r}, \bar{C}_{r}\right),\left(A_{l}, B_{l}, C_{l}\right)$, and $\left(\bar{A}_{l}, \bar{B}_{l}, \bar{C}_{l}\right)$, having the following properties:

(i) $A_{r}, B_{r}$, and $C_{r}$ are $p \times p, p \times M$, and $N \times p$ matrices, respectively, and $A_{r}$ has only eigenvalues of modulus larger than one.

(ii) $\bar{A}_{r}, \bar{B}_{r}$, and $\bar{C}_{r}$ are $\bar{p} \times \bar{p}, \bar{p} \times N$, and $M \times \bar{p}$ matrices, respectively, and $\bar{A}_{r}$ is a nonsingular matrix which has only eigenvalues of modulus less than one.

(iii) $\bar{A}_{l}, \bar{B}_{l}$, and $\bar{C}_{l}$ are $\bar{p} \times \bar{p}, \bar{p} \times m$, and $N \times \bar{p}$ matrices, respectively, and $\bar{A}_{l}$ is a nonsingular matrix which has only eigenvalues of modulus less than one.

(iv) $A_{l}, B_{l}$, and $C_{l}$ are $p \times p, p \times N$, and $M \times p$ matrices, respectively, and $A_{l}$ has only eigenvalues of modulus larger than one.

The representations $(5.5 \mathrm{a}),(5.5 \mathrm{~b})$ are reproduced by taking $A_{l}=A_{r}=\operatorname{diag}\left(\zeta_{k}\right)$ and $\bar{A}_{l}=$ $\bar{A}_{r}=\operatorname{diag}\left(\bar{\zeta}_{k}\right)$. 
Recall that the Marchenko kernels vanish for even arguments $s$. Taking $A=A_{l}=A_{r}$ and $\bar{A}=\bar{A}_{l}=\bar{A}_{r}$ and writing

$$
\begin{array}{lll}
A=\left(\begin{array}{cc}
\mathcal{A} & 0 \\
0 & -\mathcal{A}
\end{array}\right), & B_{r}=\left(\begin{array}{c}
\mathcal{B}_{r} \\
\mathcal{B}_{r}
\end{array}\right), & C_{r}=\left(\begin{array}{ll}
\mathcal{C}_{r} & \mathcal{C}_{r}
\end{array}\right), \\
\bar{A}=\left(\begin{array}{cc}
\overline{\mathcal{A}} & 0 \\
0 & -\overline{\mathcal{A}}
\end{array}\right), & \bar{B}_{r}=\left(\begin{array}{ll}
\overline{\mathcal{B}}_{r} \\
\overline{\mathcal{B}}_{r}
\end{array}\right), & \bar{C}_{r}=\left(\begin{array}{ll}
\overline{\mathcal{C}}_{r} & \overline{\mathcal{C}}_{r}
\end{array}\right),
\end{array}
$$

and similarly with the subscript $l$ instead of $r$, we obtain

$$
\begin{aligned}
& \boldsymbol{F}_{r}(s)=\hat{\boldsymbol{\rho}}(s)+\left[1+(-1)^{s+1}\right] \mathcal{C}_{r} \mathcal{A}^{-(s+1)} \mathcal{B}_{r}, \\
& \overline{\boldsymbol{F}}_{r}(s)=\hat{\overline{\boldsymbol{\rho}}}(s)+\left[1+(-1)^{s+1}\right] \overline{\mathcal{C}}_{r} \overline{\mathcal{A}}^{s-1} \overline{\mathcal{B}}_{r}, \\
& \overline{\boldsymbol{F}}_{l}(s)=\hat{\overline{\boldsymbol{\ell}}}(s)+\left[1+(-1)^{s+1}\right] \overline{\mathcal{C}}_{l} \overline{\mathcal{A}}^{-(s+1)} \overline{\mathcal{B}}_{l}, \\
& \boldsymbol{F}_{l}(s)=\hat{\boldsymbol{\ell}}(s)+\left[1+(-1)^{s+1}\right] \mathcal{C}_{l} \mathcal{A}^{s-1} \mathcal{B}_{l} .
\end{aligned}
$$

Using parity symmetry in the form (5.8a)-(5.8d), the Marchenko equations (5.6a)-(5.6d) can be decoupled further. For details we refer to Appendix B.

Let us now discuss the focusing case.

Proposition 5.1 (Symmetries of the Marchenko kernel) In the focusing case the Marchenko kernels satisfy the conjugation symmetry relations

$$
\overline{\boldsymbol{F}}_{r}(s)=-\boldsymbol{F}_{r}(s)^{\dagger}, \quad \overline{\boldsymbol{F}}_{l}(s)=-\boldsymbol{F}_{l}(s)^{\dagger} .
$$

Moreover, the Marchenko equations (5.6a)-(5.6d) are uniquely solvable.

Proof Consider the focusing case. Then (4.4) and (4.5a), (4.5b) imply that

$$
\hat{\bar{\rho}}(s)=-\hat{\boldsymbol{\rho}}(s)^{\dagger}, \quad \hat{\bar{\ell}}(s)=-\hat{\ell}(s)^{\dagger} .
$$

In the case of finitely many simple poles of the transmission coefficients, we get $\bar{\zeta}_{k}=1 / \zeta_{k}^{*}$ from (4.4). Using (5.1a), (5.1b) we get for the residues

$$
\boldsymbol{t}_{l k}=-\left[\zeta_{k}\right]^{2} \overline{\boldsymbol{t}}_{r k}^{\dagger}, \quad \boldsymbol{t}_{r k}=-\left[\zeta_{k}\right]^{2} \overline{\boldsymbol{t}}_{l k}^{\dagger}, \quad \overline{\boldsymbol{t}}_{l k}=-\left[\bar{\zeta}_{k}\right]^{2} \boldsymbol{t}_{r k}^{\dagger}, \quad \overline{\boldsymbol{t}}_{r k}=-\left[\bar{\zeta}_{k}\right]^{2} \boldsymbol{t}_{l k}^{\dagger}
$$

For the norming constants we get in a similar way

$$
\boldsymbol{C}_{l k}=-\left[\zeta_{k}\right]^{2} \overline{\boldsymbol{C}}_{r k}^{\dagger}, \quad \boldsymbol{C}_{r k}=-\left[\zeta_{k}\right]^{2} \overline{\boldsymbol{C}}_{l k}^{\dagger}, \quad \overline{\boldsymbol{C}}_{l k}=-\left[\bar{\zeta}_{k}\right]^{2} \boldsymbol{C}_{r k}^{\dagger}, \quad \overline{\boldsymbol{C}}_{r k}=-\left[\bar{\zeta}_{k}\right]^{2} \boldsymbol{C}_{l k}^{\dagger}
$$

The unique solvability of (5.6a)-(5.6d) is a standard argument $[6,9]$.

The conjugation relations (5.9) can be used to reduce the number of independent matrix triplets from four to two. In fact, in the focusing case we define

$$
\begin{array}{lll}
\overline{\mathcal{A}}=\mathcal{A}^{\dagger-1}, & \overline{\mathcal{B}}_{r}=\mathcal{A}^{\dagger-1} \mathcal{C}_{r}^{\dagger}, & \overline{\mathcal{C}}_{r}=-\mathcal{B}_{r}^{\dagger} \mathcal{A}^{\dagger-1}, \\
\overline{\mathcal{A}}=\mathcal{A}^{\dagger-1}, & \overline{\mathcal{B}}_{l}=\mathcal{A}^{\dagger-1} \mathcal{C}_{l}^{\dagger}, & \overline{\mathcal{C}}_{l}=-\mathcal{B}_{l}^{\dagger} \mathcal{A}^{\dagger-1} .
\end{array}
$$

The simplifications resulting from (5.10a), (5.10b) have been used to simplify the explicit matrix IDNLS solutions obtained in [11] in the focusing case. 
Acknowledgements F. Demontis research supported by INdAM, MIUR under PRIN grant No. 20083KLJEZ-003, and the Autonomous Region of Sardinia (RAS) under grant CRP3-138, L.R. 7/2007.

C. van der Mee research supported by RAS under grant PO Sardegna 2007-2013, L.R. 7/2007.

\section{Appendix A: Summation by Parts Formula}

Let $\left\{b_{k}\right\}_{k=n}^{\infty}$ be a sequence of nonnegative numbers. Then in [6, Lemma A.2] the following fundamental equality has been established:

$$
\sum_{k=n}^{\infty} b_{k}\left(\sum_{j=k+1}^{\infty} b_{j}\right)^{m}=\frac{1}{m+1}\left(\sum_{j=n}^{\infty} b_{j}\right)^{m+1}-B_{n}^{(m)},
$$

where $B_{n}^{(m)} \geq B_{n+1}^{(m)} \geq B_{n+2}^{(m)} \geq \cdots \geq 0$.

Although the general form of the discrete Gronwall inequality is well-known [21, Corollary 1.6.2], here we apply (A.1) to prove the version needed.

Proposition A.1 (Gronwall's inequality) Suppose $\left\{p_{k}\right\}_{k=n}^{\infty}$ and $\left\{q_{k}\right\}_{k=n}^{\infty}$ are sequences of nonnegative numbers such that the series $\sum_{k=n+1}^{\infty} q_{k}$ converges and

$$
p_{n} \leq 1+\sum_{k=n+1}^{\infty} q_{k} p_{k}
$$

Then

$$
p_{n} \leq \exp \left(\sum_{k=n+1}^{\infty} q_{k}\right)
$$

Proof Iterating (A.2) we get

$$
\begin{aligned}
p_{n} & \leq 1+\sum_{s=1}^{\infty} \sum_{k_{1}=n+1}^{\infty} q_{k_{1}} \sum_{k_{2}=k_{1}+1}^{\infty} q_{k_{2}} \sum_{k_{3}=k_{2}+1}^{\infty} q_{k_{3}} \ldots \sum_{k_{s}=k_{s-1}+1}^{\infty} q_{k_{s}} \\
& \leq 1+\sum_{s=1}^{\infty} \frac{1}{s !}\left(\sum_{k=n+1}^{\infty} q_{k}\right)^{s}=\exp \left(\sum_{k=n+1}^{\infty} q_{k}\right),
\end{aligned}
$$

where (A.1) has been used repeatedly.

\section{Appendix B: Marchenko Equations in More Detail}

In this appendix, we decouple (5.6a)-(5.6d) by using that the Marchenko kernels vanish for even values of their arguments. Equations (B.1a)-(B.3d) below have been used in [11] to derive explicit matrix IDNLS solutions by using matrix triplets to parametrize the Marchenko kernels.

Let us decouple (5.6a) and (5.6b), using that the Marchenko kernels $\boldsymbol{F}_{r}(s)$ and $\overline{\boldsymbol{F}}_{r}(s)$ vanish for even $s$. We get 


$$
\begin{aligned}
\overline{\boldsymbol{K}}^{\text {up }}(n, n+2 \sigma) \\
=\sum_{\sigma^{\prime \prime}=0}^{\infty} \overline{\boldsymbol{F}}_{r}\left(2\left[n+\sigma^{\prime \prime}\right]+1\right) \boldsymbol{F}_{r}\left(2\left[n+\sigma^{\prime \prime}+\sigma\right]+1\right) \\
\quad+\sum_{\sigma^{\prime}=1}^{\infty} \overline{\boldsymbol{K}}^{\mathrm{up}}\left(n, n+2 \sigma^{\prime}\right) \sum_{\sigma^{\prime \prime}=0}^{\infty} \overline{\boldsymbol{F}}_{r}\left(2\left[n+\sigma^{\prime}+\sigma^{\prime \prime}\right]+1\right) \boldsymbol{F}_{r}\left(2\left[n+\sigma^{\prime \prime}+\sigma\right]+1\right),
\end{aligned}
$$

$$
\begin{aligned}
\boldsymbol{K}^{\text {up }}(n, n+2 \sigma+1) & \\
= & -\overline{\boldsymbol{F}}_{r}(2[n+\sigma]+1) \\
& +\sum_{\sigma^{\prime}=0}^{\infty} \boldsymbol{K}^{\mathrm{up}}\left(n, n+2 \sigma^{\prime}+1\right) \sum_{\sigma^{\prime \prime}=1}^{\infty} \boldsymbol{F}_{r}\left(2\left[n+\sigma^{\prime}+\sigma^{\prime \prime}\right]+1\right) \overline{\boldsymbol{F}}_{r}\left(2\left[n+\sigma^{\prime \prime}+\sigma\right]+1\right),
\end{aligned}
$$

$$
\begin{aligned}
\overline{\boldsymbol{K}}^{\mathrm{dn}}(n, n+2 \sigma+1) \\
=-\boldsymbol{F}_{r}(2[n+\sigma]+1) \\
\quad+\sum_{\sigma^{\prime}=0}^{\infty} \overline{\boldsymbol{K}}^{\mathrm{dn}}\left(n, n+2 \sigma^{\prime}+1\right) \sum_{\sigma^{\prime \prime}=1}^{\infty} \overline{\boldsymbol{F}}_{r}\left(2\left[n+\sigma^{\prime}+\sigma^{\prime \prime}\right]+1\right) \boldsymbol{F}_{r}\left(2\left[n+\sigma^{\prime \prime}+\sigma\right]+1\right),
\end{aligned}
$$

$$
\begin{aligned}
& \boldsymbol{K}^{\mathrm{dn}}(n, n+2 \sigma) \\
& =\sum_{\sigma^{\prime \prime}=0}^{\infty} \boldsymbol{F}_{r}\left(2\left[n+\sigma^{\prime \prime}\right]+1\right) \overline{\boldsymbol{F}}_{r}\left(2\left[n+\sigma^{\prime \prime}+\sigma\right]+1\right) \\
& \quad+\sum_{\sigma^{\prime}=1}^{\infty} \boldsymbol{K}^{\mathrm{dn}}\left(n, n+2 \sigma^{\prime}\right) \sum_{\sigma^{\prime \prime}=0}^{\infty} \boldsymbol{F}_{r}\left(2\left[n+\sigma^{\prime}+\sigma^{\prime \prime}\right]+1\right) \overline{\boldsymbol{F}}_{r}\left(2\left[n+\sigma^{\prime \prime}+\sigma\right]+1\right) .
\end{aligned}
$$

Equations (B.1a) and (B.1d) are valid for $\sigma \geq 1$, whereas (B.1b) and (B.1c) are valid for $\sigma \geq 0$. This distinction in the ranges of the summation index $\sigma$ is to bear in mind when deriving exact solutions to (1.5). The potentials are then computed as follows:

$$
\boldsymbol{u}_{n}=\frac{i}{2 h} \boldsymbol{K}^{\mathrm{up}}(n-1, n), \quad \boldsymbol{w}_{n}=\frac{-i}{2 h} \overline{\boldsymbol{K}}^{\mathrm{dn}}(n-1, n)
$$

Let us decouple (5.6c) and (5.6d), using that the Marchenko kernels $\boldsymbol{F}_{l}(s)$ and $\overline{\boldsymbol{F}}_{l}(s)$ vanish for even $s$. We get

$$
\begin{aligned}
& \boldsymbol{L}^{\mathrm{up}}(n, n-2 \sigma) \\
& =\sum_{\sigma^{\prime \prime}=0}^{\infty} \boldsymbol{F}_{l}\left(2\left[n-\sigma^{\prime \prime}\right]-1\right) \overline{\boldsymbol{F}}_{l}\left(2\left[n-\sigma^{\prime \prime}-\sigma\right]-1\right) \\
& \quad+\sum_{\sigma^{\prime}=1}^{\infty} \boldsymbol{L}^{\mathrm{up}}\left(n, n-2 \sigma^{\prime}\right) \sum_{\sigma^{\prime \prime}=0}^{\infty} \boldsymbol{F}_{l}\left(2\left[n-\sigma^{\prime}-\sigma^{\prime \prime}\right]-1\right) \overline{\boldsymbol{F}}_{l}\left(2\left[n-\sigma^{\prime \prime}-\sigma\right]-1\right),
\end{aligned}
$$




$$
\begin{aligned}
\overline{\boldsymbol{L}}^{\mathrm{up}}(n, n-2 \sigma-1) \\
=-\boldsymbol{F}_{l}(2[n-\sigma]-1) \\
\quad+\sum_{\sigma^{\prime}=0}^{\infty} \overline{\boldsymbol{L}}^{\mathrm{up}}\left(n, n-2 \sigma^{\prime}-1\right) \sum_{\sigma^{\prime \prime}=1}^{\infty} \overline{\boldsymbol{F}}_{l}\left(2\left[n-\sigma^{\prime}-\sigma^{\prime \prime}\right]-1\right) \boldsymbol{F}_{l}\left(2\left[n-\sigma^{\prime \prime}-\sigma\right]-1\right),
\end{aligned}
$$

$$
\begin{aligned}
\boldsymbol{L}^{\mathrm{dn}}(n, n-2 \sigma-1) \\
=-\overline{\boldsymbol{F}}_{l}(2[n-\sigma]-1) \\
\quad+\sum_{\sigma^{\prime}=0}^{\infty} \boldsymbol{L}^{\mathrm{dn}}\left(n, n-2 \sigma^{\prime}-1\right) \sum_{\sigma^{\prime \prime}=1}^{\infty} \boldsymbol{F}_{l}\left(2\left[n-\sigma^{\prime}-\sigma^{\prime \prime}\right]-1\right) \overline{\boldsymbol{F}}_{l}\left(2\left[n-\sigma^{\prime \prime}-\sigma\right]-1\right),
\end{aligned}
$$

$$
\begin{aligned}
\overline{\boldsymbol{L}}^{\mathrm{dn}} & (n, n-2 \sigma) \\
= & \sum_{\sigma^{\prime \prime}=0}^{\infty} \overline{\boldsymbol{F}}_{l}\left(2\left[n-\sigma^{\prime \prime}\right]-1\right) \boldsymbol{F}_{l}\left(2\left[n-\sigma^{\prime \prime}-\sigma\right]-1\right) \\
& +\sum_{\sigma^{\prime}=1}^{\infty} \overline{\boldsymbol{L}}^{\mathrm{dn}}\left(n, n-2 \sigma^{\prime}\right) \sum_{\sigma^{\prime \prime}=0}^{\infty} \overline{\boldsymbol{F}}_{l}\left(2\left[n-\sigma^{\prime}-\sigma^{\prime \prime}\right]-1\right) \boldsymbol{F}_{l}\left(2\left[n-\sigma^{\prime \prime}-\sigma\right]-1\right) .
\end{aligned}
$$

Equations (B.3a) and (B.3d) are valid for $\sigma \geq 1$, whereas (B.3b) and (B.3c) are valid for $\sigma \geq 0$. This distinction in the ranges of the summation index $\sigma$ is to bear in mind when deriving exact solutions of (1.5). The potentials are then computed as follows:

$$
\boldsymbol{u}_{n}=\frac{i}{2 h} \overline{\boldsymbol{L}}^{\mathrm{up}}(n+1, n), \quad \boldsymbol{w}_{n}=\frac{-i}{2 h} \boldsymbol{L}^{\mathrm{dn}}(n+1, n) \text {. }
$$

\section{References}

1. Ablowitz, M.J., Biondini, G., Prinari, B.: Inverse scattering transform for the integrable nonlinear Schrödinger equation with nonvanishing boundary conditions. Inverse Probl. 23, 1711-1758 (2007)

2. Ablowitz, M.J., Ladik, J.F.: Nonlinear differential-difference equations. J. Math. Phys. 16, 598-603 (1975)

3. Ablowitz, M.J., Ladik, J.F.: A nonlinear difference scheme and inverse scattering. Stud. Appl. Math. 55, 213-229 (1976)

4. Ablowitz, M.J., Ladik, J.F.: Nonlinear differential-difference equations and Fourier analysis. J. Math. Phys. 17, 1011-1018 (1976)

5. Ablowitz, M.J., Ohta, Y., Trubatch, A.D.: On discretizations of the vector nonlinear Schrödinger equation. Phys. Lett. A 253, 287-304 (1999)

6. Ablowitz, M.J., Prinari, B., Trubatch, A.D.: Discrete and Continuous Nonlinear Schrödinger Systems. London Math. Soc. Lecture Notes Series, vol. 302. Cambridge Univ. Press, Cambridge (2004)

7. Cai, D., Bishop, A.P., Grønbech-Jensen, N.: Spatially localized, temporally quasi-periodic, discrete nonlinear excitations. Phys. Rev. E 52, 5784-5787 (1995)

8. Deift, P., Trubowitz, E.: Inverse scattering on the line. Commun. Pure Appl. Math. 32, 121-251 (1979)

9. Demontis, F.: Matrix Zakharov-Shabat System and Inverse Scattering Transform. Lambert Academic, Saarbrücken (2012). Also: F. Demontis, Direct and Inverse Scattering of the Matrix Zakharov-Shabat System, Ph.D. thesis, University of Cagliari, Italy, 2007

10. Demontis, F., van der Mee, C.: Exact solutions to the integrable discrete nonlinear Schrödinger equation under a quasiscalarity condition. Commun. Appl. Ind. Math. 2(2), e-372 (2011), 21 pp. 
11. Demontis, F., van der Mee, C.: Closed form solutions to the integrable discrete nonlinear Schrödinger equation. J. Nonlinear Math. Phys. 19(2), 1250010 (2012), 22 pp.

12. Doliwa, A., Santini, P.M.: Integrable dynamics of a discrete curve and the Ablowitz-Ladik hierarchy. J. Math. Phys. 36, 1259-1273 (1995)

13. Faddeev, L.D.: Properties of the $S$ - matrix of the one-dimensional Schrödinger equation. Transl. Am. Math. Soc. 2, 139-166 (1964); also: Trudy Mat. Inst. Steklova 73, 314-336 (1964) [Russian]

14. Gerdjikov, V.S., Ivanov, M.I.: Block discrete Zakharov-Shabat system. I. Generalized Fourier expansion, United Nuclear Research Institute Report E2-81-811, Dubna, 1981

15. Gerdjikov, V.S., Ivanov, M.I.: Block discrete Zakharov-Shabat system. I. Hamiltonian structures, United Nuclear Research Institute Report E2-81-812, Dubna, 1981

16. Hasegawa, A., Matsumoto, M.: Optical Solitons in Fibers, 3rd edn. Springer, Berlin (2002)

17. Hasegawa, A., Tappert, F.: Transmission of stationary nonlinear optical pulses in dispersive dielectric fibers. I. Anomalous dispersion. Appl. Phys. Lett. 23, 142-144 (1973)

18. Hisakado, M., Wadati, M.: Integrable dynamics of discrete surfaces. II. J. Phys. Soc. Jpn. 65, 389-393 (1996)

19. Klaus, M.: Low-energy behaviour of the scattering matrix for the Schrödinger equation on the line. Inverse Probl. 4, 505-512 (1988)

20. Kulish, P.P.: Quantum difference nonlinear Schrödinger equation. Lett. Math. Phys. 5, 191-197 (1981)

21. Lakshmikantham, V., Trigiante, D.: Theory of Difference Equations: Numerical Methods and Applications. Mathematics in Science and Engineering, vol. 181. Academic Press, New York (1988)

22. Manakov, S.V.: On the theory of two-dimensional stationary self-focusing of electromagnetic waves. Sov. Phys. JETP 38, 248-253 (1974)

23. Suris, Y.B.: A note on an integrable discretization of the nonlinear Schrödinger equation. Inverse Probl. 13, 1121-1136 (1997)

24. Tsuchida, T., Ujino, H., Wadati, M.: Integrable semi-discretization of the coupled nonlinear Schrödinger equations. J. Phys. A 32, 2239-2262 (1999)

25. Vekslerchik, V.E., Konotop, V.V.: Discrete nonlinear Schrödinger equation under non-vanishing boundary conditions. Inverse Probl. 8, 889-909 (1992)

26. Wiener, N.: The Fourier Integral and Certain of Its Applications. Dover, New York (1959); also: Cambridge Univ. Press, Cambridge (1933)

27. Zakharov, V.E., Shabat, A.B.: Exact theory of two-dimensional self-focusing and one dimensional selfmodulation of waves in nonlinear media. Sov. Phys. JETP 34, 62-69 (1972) 